\title{
THE CLUSTER LENSING AND SUPERNOVA SURVEY WITH HUBBLE: AN OVERVIEW
}

\author{
Marc Postman $^{1}$, Dan Coe ${ }^{1}$, Narciso Benítez ${ }^{2}$, Larry Bradley ${ }^{1}$, Tom Broadhurst ${ }^{3}$, Megan Donahue $^{4}$, Holland Ford ${ }^{5}$, \\ Or Graur $^{6}$, Genevieve Graves ${ }^{7}$, Stephanie Jouvel $^{8}$, Anton KoeKemoer ${ }^{1}$, Doron Lemze ${ }^{5}$, Elinor Medezinski ${ }^{5}$, \\ Alberto Molino $^{2}$, Leonidas Moustakas $^{9}$, Sara Ogaz $^{1}$, Adam Riess $^{1,5}$, Steve Rodney ${ }^{5}$, Piero Rosati ${ }^{10}$, Keitchi Umetsu $^{11}$, \\ Wei Zheng ${ }^{5}$, Adi Zitrin ${ }^{6}$, Matthias Bartelmann ${ }^{12}$, Rychard Bouwens ${ }^{13}$, Nicole Czakon ${ }^{8}$, Sunil Golwala $^{8}$, Ole Host ${ }^{14}$, \\ LeOpoldo Infante $^{15}$, SAurabh JhA ${ }^{16}$, Yolanda Jimenez-Teja ${ }^{2}$, Daniel Kelson ${ }^{17}$, Ofer Lahav ${ }^{14}$, Ruth LazKOZ ${ }^{3}$, \\ Dani Maoz ${ }^{6}$, Curtis McCully ${ }^{16}$, Peter Melchior ${ }^{18}$, Massimo Meneghetti $^{19}$, Julian Merten ${ }^{12}$, John Moustakas $^{20}$, \\ Mario Nonino ${ }^{21}$, Brandon Patel ${ }^{16}$, Enikö Regös ${ }^{22}$, Jack Sayers ${ }^{8}$, Stella Seitz ${ }^{23}$, and Arjen Van der Wel ${ }^{24}$ \\ ${ }^{1}$ Space Telescope Science Institute, 3700 San Martin Drive, Baltimore, MD 21208, USA; postman@ stsci.edu \\ ${ }^{2}$ Instituto de Astrofísica de Andalucía (CSIC), C/Camino Bajo de Huétor 24, Granada 18008, Spain \\ ${ }^{3}$ Department of Theoretical Physics, University of the Basque Country, P. O. Box 644, 48080 Bilbao, Spain \\ ${ }^{4}$ Department of Physics and Astronomy, Michigan State University, East Lansing, MI 48824, USA \\ ${ }^{5}$ Department of Physics and Astronomy, The Johns Hopkins University, 3400 North Charles Street, Baltimore, MD 21218, USA \\ ${ }^{6}$ School of Physics and Astronomy, Tel Aviv University, Tel-Aviv 69978, Israel \\ ${ }^{7}$ Department of Astronomy, University of California, 601 Campbell Hall, Berkeley, CA 94720, USA \\ 8 Jet Propulsion Laboratory, California Institute of Technology, MS 169-327, Pasadena, CA 91109, USA \\ ${ }^{9}$ ESO-European Southern Observatory, D-85748 Garching bei München, Germany \\ ${ }^{10}$ Institute of Astronomy and Astrophysics, Academia Sinica, P. O. Box 23-141, Taipei 10617, Taiwan \\ ${ }^{11}$ Institut für Theoretische Astrophysik, ZAH, Albert-Ueberle-Straß e 2, 69120 Heidelberg, Germany \\ ${ }^{12}$ Leiden Observatory, Leiden University, P. O. Box 9513,2300 RA Leiden, The Netherlands \\ ${ }^{13}$ Department of Physics, Mathematics, and Astronomy, California Institute of Technology, Pasadena, CA91125, USA \\ ${ }^{14}$ Department of Physics \& Astronomy. University College London, Gower Street, London WCIE 6 BT, UK \\ ${ }^{15}$ Departamento de Astronoía y Astrofísica, Pontificia Universidad Católica de Chile, V. Mackenna 4860, Santiago 22, Chile \\ ${ }^{16}$ Department of Physics and Astronomy, Rutgers University, 136 Frelinghuysen Rd., Piscataway, NJ 08854, USA \\ 17 Observatories of the Carnegie Institution of Washington, Pasadena, CA 91 101, USA \\ ${ }^{18}$ Center for Cosmology and Astro-Particle Physics, \& Department of Physics; The Ohio State University, 191 W. Woodruff Ave., Columbus, Ohio 43210, USA \\ 19 INAF, Osservatorio Astronomico di Bologna, \& INFN, Sezione di Bologna; Via Ranzani 1, I-40127 Bologna, Italy \\ ${ }^{20}$ Center for Astrophysics and Space Sciences, University of California at San Diego. 9500 Gilman Dr., MC 0424, La Jolla, CA 92093, USA \\ ${ }^{21}$ INAF-Osservatorio Astronomico di Trieste, via G.B. Tiepolo 11, 40131 Trieste, Italy \\ 22 European Laboratory for Particle Physics (CERN). CH-1211, Geneva 23, Switzerland \\ ${ }^{23}$ Universitäts-Sternwarte, München, Scheinerstr. 1, D-81679 München. Germany \\ ${ }^{24}$ Max-Planck Institute for Astronomy, Königstuhl 17, D-69117, Heidelberg, Germany \\ Received 2011 June 16; accepted 2011 December 5; published 2012 March 14
}

\begin{abstract}
The Cluster Lensing And Supernova survey with Hubble (CLASH) is a 524-orbit Multi-Cycle Treasury Program to use the gravitational lensing properties of 25 galaxy clusters to accurately constrain their mass distributions. The survey, described in detail in this paper, will definitively establish the degree of concentration of dark matter in the cluster cores, a key prediction of structure formation models. The CLASH cluster sample is larger and less biased than current samples of space-based imaging studies of clusters to similar depth, as we have minimized lensing-based selection that favors systems with overly dense cores. Specifically, 20 CLASH clusters are solely $\mathrm{X}$-ray selected. The X-ray-selected clusters are massive $(k T>5 \mathrm{keV})$ and, in most cases, dynamically relaxed. Five additional clusters are included for their lensing strength $\left(\theta_{\text {Ein }}>35^{\prime \prime}\right.$ at $\left.z_{s}=2\right)$ to optimize the likelihood of finding highly magnified high $-z(z>7)$ galaxies. A total of 16 broadband filters, spanning the near-UV to near-IR, are employed for each 20-orbit campaign on each cluster. These data are used to measure precise $\left(\sigma_{z} \sim 0.02(1+z)\right)$ photometric redshifts for newly discovered arcs. Observations of each cluster are spread over eight epochs to enable a search for Type Ia supernovae at $z>1$ to improve constraints on the time dependence of the dark energy equation of state and the evolution of supernovae. We present newly re-derived X-ray luminosities, temperatures, and Fe abundances for the CLASH clusters as well as a representative source list for MACS1149.6+2223 $(z=0.544)$.
\end{abstract}

Key words: dark energy - dark matter - Galaxy: evolution - Galaxy: formation - gravitational lensing: strong gravitational lensing: weak

Online-only material: color figures, machine-readable table

\section{INTRODUCTION}

The universe has proven to be far more intriguing in its composition than we knew it to be even just 14 years ago. It is a "dark" universe where $\sim 23 \%$ of its mass-energy density is made up of weakly interacting (and, as yet, undetected) nonbaryonic particles (a.k.a. dark matter, DM) and $\sim 73 \%$ is as yet unknown physics (a.k.a. dark energy) that is driving an accelerated expansion of the metric (e.g., Komatsu et al. 2011;
Riess et al. 2011). The Hubble Space Telescope (HST) has played a key role in providing evidence for and constraining the nature of both of these mysterious dark components (e.g., Riess et al. 1998; Perlmutter et al. 1999; Clowe et al. 2006).

Clusters of galaxies, by virtue of their position at the high end of the cosmic mass power spectrum, provide a powerful way to constrain the frequency of high amplitude perturbations in the primordial density field. As such, they play a direct and fundamental role in testing cosmological models and in constraining 
the properties of DM, providing unique and independent tests of any viable cosmology and structure formation scenario, and possible modifications of the laws of gravity. A key ingredient of such cluster-based cosmological tests is the mass distribution of clusters, both on (sub) Mpc scales and across the range of populations. The best and highest resolution maps of DM distribution in massive galaxy clusters come from observations of strong gravitational lensing made by the Advanced Camera for Surveys (ACS; Ford et al. 2003) on board HST. As part of a Guaranteed Time Observation (GTO) program, deep (20orbit) multiband (4-6 filter) observations were obtained for five galaxy clusters: A1689 (Broadhurst et al. 2005; Limousin et al. 2007; Coe et al. 2010), A1703 (Limousin et al. 2008; Saha \& Read 2009; Zitrin et al. 2010), A2218 (Elíasdóttir et al. 2007), CL0024+1654 (Jee et al. 2007; Zitrin et al. 2009; Umetsu et al. 2010), and MS1358+6245 (Zitrin et al. 2011c). These results have contributed to a reported tension between observed and simulated galaxy cluster DM halos. Observed clusters appear to have denser cores than simulated clusters of similar total (virial) mass (e.g., Broadhurst et al. 2008; Oguri et al. 2009; Sereno et al. 2010). Understanding the true constraints from observed concentration and mass profile measurements on $\Lambda \mathrm{CDM}$ structure formation models is one of the important problems that can be tackled with a deep, high angular resolution imaging survey of a significantly larger and more homogeneously selected sample of galaxy clusters.

In 2009 May, NASA successfully executed the final planned Hubble Servicing mission, SM4, with the installation of the Wide Field Camera 3 (WFC3; Kimble et al. 2008) and the repair of ACS. Shortly thereafter, the Hubble Multi-Cycle Treasury (MCT) Program was conceived to permit ambitious programs ( $>500$ orbits) with broad scientific potential that could not be accomplished within the constraints of a single HST observing cycle and that would take full advantage of the final era of a newly refurbished $H S T$.

The Cluster Lensing and Supernova survey with Hubble (CLASH) was one of three MCT programs selected. CLASH has four main science goals.

1. Measure the profiles and substructures of DM in galaxy clusters with unprecedented precision and resolution.

2. Detect Type Ia supernovae (SNe Ia) out to redshift $z \sim 2.5$ to measure the time dependence of the dark energy equation of state and potential evolutionary effects in the SNe themselves.

3. Detect and characterize some of the most distant galaxies yet discovered at $z>7$.

4. Study the internal structure and evolution of the galaxies in and behind these clusters.

To accomplish these objectives, the CLASH program targets 25 massive galaxy clusters and will image each in 16 passbands using WFC3/UVIS, WFC3/IR, and ACS/WFC. CLASH has been allocated $524 H S T$ orbits, spread out over cycles 18, 19, and 20. The majority of these orbits (474) are for cluster imaging and, simultaneously, for the parallel SN search program. An additional 50 orbits were allocated as a reserve for SN followup observations. Based on a current census of the HST data archive, CLASH will produce a six-fold increase in the number of lensing clusters observed to a depth of 20 orbits and, more importantly, will vastly increase the number of lensing clusters with extensive multiband HST imaging.

Motivations for each of the main CLASH science goals are provided in Sections 2.1-2.4. Subsequent sections describe the cluster sample (Section 3), survey design (Section 4), data pipeline (Section 5), and supporting observations using other facilities (Section 6). Data products intended for public distribution to the community are briefly described in Section 7. AB magnitudes are used throughout (Oke 1974). The cosmologi-

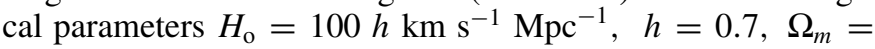
0.30 , and $\Lambda=0.70$ are assumed in this paper.

\section{SCIENTIFIC MOTIVATION}

\subsection{Galaxy Cluster Dark Matter Profiles and Formation Times}

Recent observations suggest that galaxy clusters formed earlier in our universe than in simulated $\Lambda \mathrm{CDM}$ universes. These observations include the detection of perhaps unexpectedly massive galaxy clusters at $z>1$ (Stanford et al. 2006; Eisenhardt et al. 2008; Jee et al. 2009, 2011; Huang et al. 2009; Rosati et al. 2009; Papovich et al. 2010; Schwope et al. 2010; Gobat et al. 2011; Foley et al. 2011) and the finding that some clusters at intermediate redshift $(z \sim 0.3)$ have denser cores than clusters of similar mass produced in simulations (Broadhurst et al. 2008; Broadhurst \& Barkana 2008; Oguri et al. 2009; Richard et al. 2010; Sereno et al. 2010; Zitrin et al. 2011a). While the evidence to date for early cluster growth is suggestive, possible explanations include departures from the Gaussian initial density fluctuation spectrum or higher levels of dark energy in the past, so-called Early Dark Energy (EDE; Fedeli \& Bartelmann 2007; Sadeh \& Rephaeli 2008; Francis et al. 2009; Grossi \& Springel 2009). If a significant quantity of EDE (for example, $\Omega_{\mathrm{DE}} \sim 0.1$ at $z=6$ ) suppressed structure growth in the early universe, then clusters would have had to start forming sooner to yield the numbers we observe today. These scenarios remain allowable within current observational constraints as described in the above papers, although some nonGaussian models can be ruled out by using the cosmic X-ray background measurements (Lemze et al. 2009). The CLASH data permit significant advances to be made toward supporting or rejecting observational evidence for early cluster growth by measuring core densities for a larger, less biased sample of clusters.

In cosmological simulations, cold-dark-matter-(CDM)dominated halos of all masses consistently evolve to have a roughly "universal" density profile that steepens with radius. Functional forms that fit such a profile well include the "NFW" profile (Navarro et al. 1996, 1997) and the Einasto/Sérsic profile (Sérsic 1963; Einasto 1965; Navarro et al. 2004, 2010). Furthermore, each simulated halo's core density is related to the background density of the universe at the halo's formation time. Halos that form later, including the most massive galaxy clusters, are found to have the least dense cores in a relative sense. Determining the relationship between the shape and depth of a halo's gravitational potential and its total mass as a function of time thus provides fundamental constraints on structure formation.

In practice, the relative core densities, or "concentrations," are measured (both in simulated and observed halos) as $c_{\mathrm{vir}}=$ $r_{\text {vir }} / r_{-2}$, a ratio between the virial radius and the inner radius at which the density slope of the fitted profile is isothermal $\left(\rho \propto r^{-2}\right)$. Analyses of gravitational lensing spanning such a large range of radii allow one to map the (primarily dark) matter profiles of observed halos and measure their concentrations.

DM profiles are best mapped in cluster cores using stronglensing analysis of multiband HST imaging. The lensingbased mass profile mapping is extended to the virial radii of 


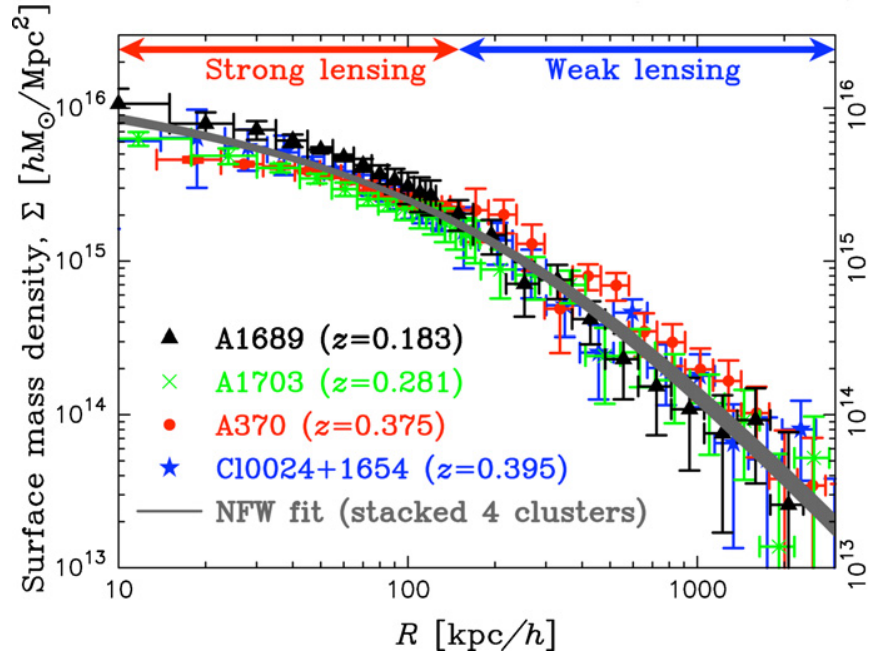

Figure 1. Mass profiles measured for four well-studied, strongly lensing galaxy clusters that are not included in the CLASH sample. All have similar mass profiles as measured from Hubble observations of strong-lensing and Subaru observations of weak-lensing distortion and magnification (Umetsu et al. 2011a, their Figure 6). The averaged mass profile is in remarkably good agreement with the standard NFW form (Umetsu et al. 2011b, their Figure 1) as shown by the gray area ( $2 \sigma$ confidence interval of the NFW fit), though with a higher concentration than predicted from cosmological simulations. Both strong- and weak-lensing probes are required to map the continuously steepening mass profile from the inner core $\left(\sim 10 \mathrm{kpc} h^{-1}\right)$ out to beyond the virial radius $\left(\sim 2 \mathrm{Mpc} h^{-1}\right)$.

(A color version of this figure is available in the online journal.)

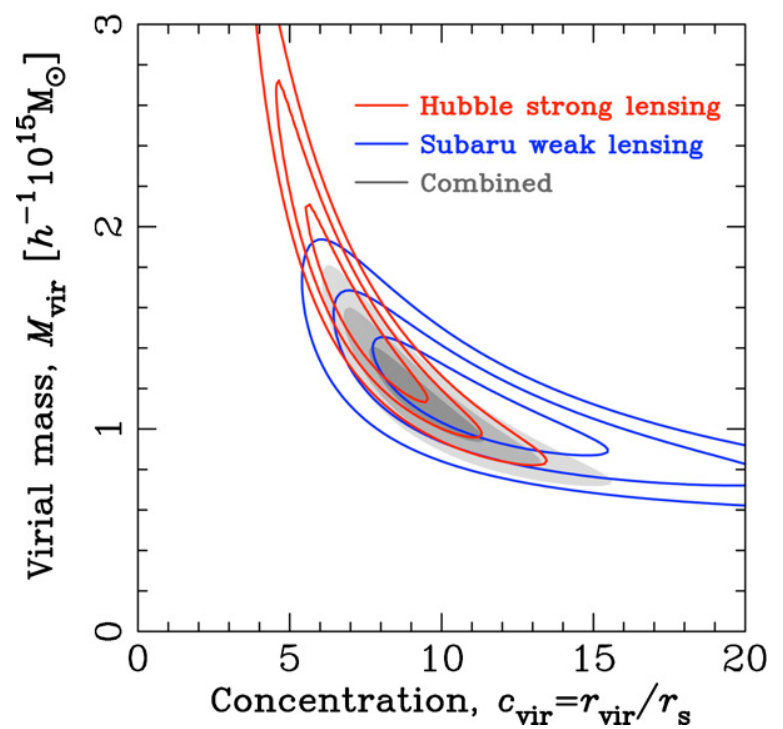

Figure 2. Joint strong- and weak-lensing analyses are required to obtain tight constraints on cluster concentrations as shown here for CL0024+17, a nonCLASH cluster (Umetsu et al. 2010, from their Figures 15 and 17). Confidence levels of $68.3 \%, 95.4 \%$, and $99.7 \%$ are plotted in the $c_{\mathrm{vir}}-M_{\mathrm{vir}}$ plane.

(A color version of this figure is available in the online journal.)

clusters using weak-lensing analysis of wider field ground-based multiband imaging, such as from Subaru. Results from Umetsu et al. (2011a, 2011b) for four of the currently best-studied (nonCLASH) clusters are shown in Figure 1.

Joint modeling of strong and weak lensing (SL+WL) yields significantly better constraints on concentrations than either probe alone. Quantitatively, Meneghetti et al. (2010) found their joint SL+WL analyses of simulated clusters yield concentrations to $\sim 11 \%$ accuracy, while WL-only and SL-only analyses yielded $\sim 33 \%$ and $\sim 59 \%$ scatters, respectively. Figure 2

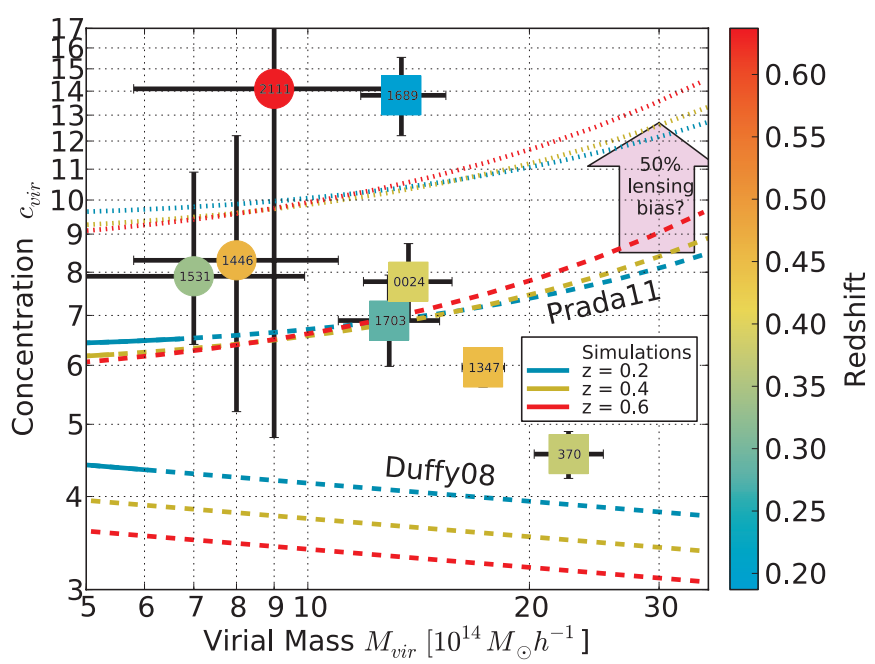

Figure 3. Mass profiles of the best-studied clusters to date are revealed to have higher central density concentrations than simulated clusters of similar mass and redshift. Reconciliation may be within reach given results from the latest simulations and an estimated lensing bias which CLASH will avoid. The plotted lines are mean concentrations at three different redshifts for clusters in these simulations as calculated from the fitting formulae provided in those papers. However, note that halos of this great mass are rare (or even non-existent at these redshifts) in these simulations, so these results are mainly extrapolations, as designated by the dashed lines. The thinner dashed lines above illustrate a $50 \%$ observational bias applied to the Prada et al. (2011) results. This bias has been roughly estimated for non-CLASH clusters such as these which were selected for study based on exceptional lensing strength (Hennawi et al. 2007; Oguri \& Blandford 2009; Meneghetti et al. 2010, 2011). Results from the currently beststudied clusters are plotted here as squares (Umetsu et al. 2011a) and circles (Oguri et al. 2009) labeled with abbreviated names and described further in Table 1.

(A color version of this figure is available in the online journal.)

demonstrates how SL and WL analyses combine to yield robust constraints on the mass and concentration of CLO024+17 (Umetsu et al. 2010).

The best-studied (SL+WL) galaxy clusters to date have been found to have overly high concentrations (dense cores) compared to halos in $\mathrm{N}$-body simulations with similar masses, as shown in Figure 3 (Broadhurst et al. 2008; Oguri et al. 2009; Sereno et al. 2010) and detailed in Table 1. Recent simulations (Prada et al. 2011) yield cluster concentrations that are over 50\% higher than previous simulations (e.g., Duffy et al. 2008). This is a product of upturns as a function of both mass and redshift found in these newer simulations, which are not yet understood. Similarly, clusters have also been found to have somewhat larger than expected Einstein radii, a direct and particularly accurate measure of the projected mass in a halo's core (Broadhurst \& Barkana 2008; Richard et al. 2010; Zitrin et al. 2011a, 2011b).

Unfortunately, the best-studied clusters to date have also been among the strongest gravitational lenses known. Such lensing-selected clusters are highly biased toward halos with high concentrations, both intrinsically and as projected on the sky due to halo elongation along the line of sight (Hennawi et al. 2007; Oguri \& Blandford 2009; Meneghetti et al. 2010, 2011). These biases are estimated to lead to systematically higher concentrations by as much as $50 \%$ or more. However, such a bias is insufficient to account for the discrepancy between the observations and the predictions, that is until a very recent analysis of new simulations was performed (see Figure 3 and discussion below).

More robust conclusions require analysis of a larger, unbiased cluster sample. Progress toward this goal has been made by 
Table 1

Concentration Measurements for Previously Well-studied Lensing-selected Clusters

\begin{tabular}{|c|c|c|c|c|c|c|}
\hline Constraints $^{\mathrm{a}}$ & Publication & Cluster & $z$ & $\begin{array}{c}M_{\mathrm{vir}} \\
\left(10^{15} M_{\odot} h^{-1}\right)\end{array}$ & $c_{\mathrm{vir}}$ & $\chi^{2} /$ dof \\
\hline $\mathrm{SL}+\mathrm{WL}+\mathrm{mag}$ & Umetsu et al. $(2011 a)^{b}$ & A1689 & 0.187 & $1.34_{-0.16}^{+0.20}$ & $\begin{array}{l}13.82_{-1.62}^{+1.72} \\
\end{array}$ & $4.73 / 17$ \\
\hline $\mathrm{SL}+\mathrm{WL}+\mathrm{mag}$ & Umetsu et al. $(2011 a)^{b}$ & A1703 & 0.281 & $1.29_{-0.19}^{+0.22}$ & $6.89_{-0.91}^{+1.04}$ & $7.14 / 19$ \\
\hline $\mathrm{SL}+\mathrm{WL}+\mathrm{mag}$ & Umetsu et al. $(2011 a)^{b}$ & A370 & 0.375 & $2.26_{-0.23}^{+0.26}$ & $4.56 \pm 0.33$ & $14.07 / 24$ \\
\hline $\mathrm{SL}+\mathrm{WL}+\mathrm{mag}$ & Umetsu et al. $(2011 a)^{b}$ & CL0024+17 & 0.395 & $1.37_{-0.18}^{+0.20}$ & $7.77_{-0.87}^{+0.97}$ & $11.47 / 20$ \\
\hline $\mathrm{SL}+\mathrm{WL}+\mathrm{mag}$ & Umetsu et al. $(2011 a)^{b}$ & RXJ1347.5-1145 & 0.451 & $1.73_{-0.11}^{+0.12}$ & $5.96_{-0.35}^{+0.37}$ & $45.06 / 25$ \\
\hline $\mathrm{RE}+\mathrm{WL}$ & Oguri et al. (2009) & SDSS J1531+3414 & 0.335 & $0.7_{-0.24}^{+0.29}$ & $7.9_{-1.5}^{+3.0}$ & $8.1 / 6$ \\
\hline $\mathrm{RE}+\mathrm{WL}$ & Oguri et al. (2009) & SDSS J1446+3032 & 0.464 & $0.8_{-0.22}^{+0.3}$ & $8.3_{-3.1}^{+3.9}$ & $6.4 / 6$ \\
\hline $\mathrm{RE}+\mathrm{WL}$ & Oguri et al. (2009) & SDSS J2111-0115 & 0.637 & $0.9_{-0.32}^{+0.41}$ & $14.1_{-9.3}^{+25.9}$ & $7.5 / 6$ \\
\hline
\end{tabular}

Notes. Of these eight clusters, only one-RXJ1347.5-1145-is in the CLASH sample.

${ }^{a} \mathrm{SL}=$ strong lensing; $\mathrm{WL}=$ weak lensing; $\mathrm{mag}=$ magnification bias (number counts); $\mathrm{RE}=$ Einstein radius.

b The parameters here are from standard NFW fits to profiles in Umetsu et al. (2011a), and not from the generalized NFW (gNFW) fits given in that paper.

LoCuSS, the Local Cluster Substructure Survey (Smith et al. 2005). A large sample of 165 clusters between $0.15<z<0.30$ was selected based on X-ray brightness. Strong-lensing analyses of 20 of these based on HST imaging (mostly single-band "snapshots") were presented by Richard et al. (2010). Okabe et al. (2010a) published weak-lensing analyses of 30 LoCuSS clusters, 22 of these being more "secure" based on multiband Subaru imaging, and 9 of these 22 overlapping with the Richard et al. (2010) subset. Subaru images are especially desirable for WL studies as they enable excellent galaxy shape measurements to be performed over a wide area. Multiband imaging is also critical to properly select background galaxies and avoid significantly diluting the weak-lensing signal (and thus the virial mass and concentration) with unlensed foreground galaxies (Medezinski et al. 2007, 2010). Stacked WL-only analyses have been performed on large cluster samples (Johnston et al. 2007; Mandelbaum et al. 2008), but we re-emphasize the need for combining strong + weak lensing analyses in addition to crosscomparisons with mass profile estimates from other techniques. For example, mass concentrations can be measured from Xray profiles (Buote et al. 2007; Ettori et al. 2010), although these are subject to uncertainties due to assumptions about hydrostatic equilibrium. Importantly, cluster elongation along the line of sight (a potential bias in concentration measurements) can be measured by the combination of lensing and X-ray analysis (Morandi et al. 2011; Newman et al. 2011). The caustic technique (Diaferio \& Geller 1997; Rines \& Diaferio 2006) is, like lensing-based methods, independent of the dynamical state of the cluster and also provides an important cross check on mass estimates from lensing and gas kinematics. Further discussion of some of the previous results from various methods is given in Comerford \& Natarajan (2007), Coe (2010), Rines et al. (2010), and King \& Mead (2011).

Reconciliation of high observed concentrations with results from simulations may ultimately come from significantly reducing the observational sample bias along with finding higher concentrations in simulated clusters. Baryons, for example, are currently absent from those cosmological simulations large enough to produce massive clusters. Baryons can result in significant "adiabatic contraction" on galaxy scales, however they constitute a much smaller fraction of the mass on cluster scales. Simulations including baryons show that cluster halo concentrations are likely only varied by $\sim 10 \%$ or so relative to DM-only halos, and that the direction of variation (increase or decrease) is not even clear, depending on the gas physics assumed (Duffy et al. 2010; Mead et al. 2010; King \& Mead 2011). Nonetheless, measuring the velocity dispersion of the brightest cluster galaxy (BCG) as an additional constraint on the inner ( $\lesssim 80 \mathrm{kpc})$ mass profile, where its stellar mass is a non-negligible component of the matter distribution, provides for a more thorough mapping of the total mass profile. Some clusters have been shown to have inner mass profiles that are shallower than NFW (Sand et al. 2004, 2008; Newman et al. 2009, 2011). Deviations of cluster mass profiles from NFW or Einasto forms at small and/or large radii may slightly bias concentration measurements (Oguri \& Hamana 2011). The use of multiple probes of the matter distribution, as CLASH is designed to do, will enable such biases to be measured and the significance of deviations determined.

More recently, an analysis by Prada et al. (2011) found that clusters in the Bolshoi and MultiDark simulations have concentrations $\sim 50 \%$ higher than clusters in previous simulations. While the robustness of this new result is still being assessed, it raises the possibility that the combination of new observations of an unbiased sample of clusters and new simulations may be able to bridge the concentration gap. We stress here that estimates of the observational bias from previous cluster studies have large uncertainties and likely vary for each cluster. CLASH will determine mass profiles and concentrations for a new cluster sample free of lensing selection bias. As we demonstrate in Section 3.2, CLASH is designed to detect (or rule out) with $99 \%$ statistical confidence average deviations of $15 \%$ or more from predicted concentrations. However, given the outstanding uncertainties in the expected concentrations, we prefer to recast the problem as follows: CLASH will deliver robust observational concentration measurements for a sample of clusters that simulations will be tasked to reproduce. Ultimately, this will lead to a better calibration of many mass estimation techniques and, consequently, to a better understanding of structure formation on cluster scales and perhaps of our cosmological model as well.

\subsection{Improved Constraints on the Dark Energy Equation of State and SNe Evolution}

The biggest cosmological surprise in decades came from observations of high-redshift SNe Ia, providing the first evidence that the expansion of the universe now appears to be accelerating (Riess et al. 1998; Perlmutter et al. 1999), and indicating that the universe is dominated by "dark energy." The presence of dark 


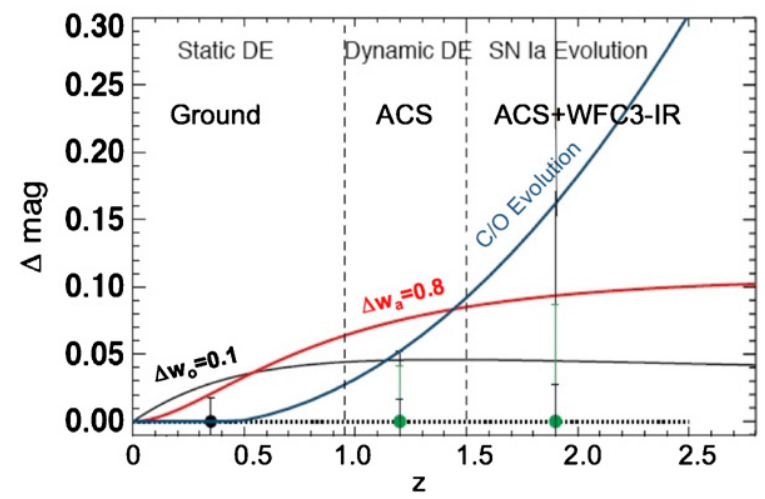

Figure 4. Dark energy and evolution sensitivity. Three models consistent with current data are shown: $w_{0}=0.9, w_{a}=0$ (black); $w_{0}=1, w_{a}=0.8$ (red); and $w_{0}=1, w_{a}=0$ (dashed). Also plotted blue is the SNe Ia evolution model of Domínguez et al. (2001), where the peak luminosity changes 3\% per solar mass change in the donor star. The error bars show the constraints at present, as projected after CLASH, and if HST continues to collect SNe Ia at the present rate for seven years.

(A color version of this figure is available in the online journal.)

energy has galvanized cosmologists as they seek to understand it. Observations of high-redshift SNe Ia have continued to lead the way in measuring the properties of dark energy (e.g., Riess et al. 2011). The goal for cosmologists now is to measure the equation of state of dark energy, $w=P /\left(\rho c^{2}\right)$, and its time variation in the hope of discriminating between viable explanations. A departure of the present equation of state, $w_{0}$, from -1 or a detection of its variation, $\partial w / \partial z$, would invalidate an innate vacuum energy (i.e., the cosmological constant) as the source of dark energy and would point toward a present epoch of "weak inflation." A difference between the expansion history and the growth history of structure expected for $w(z)$ would point toward a breakdown in general relativity as the cosmic scale factor approaches unity.

$H S T$ paired with ACS is a unique tool in this investigation, providing the only means to collect $\mathrm{SNe}$ Ia at $1<z<1.5$, which, in turn, provide the only constraints we have to date on the time variation of $w$. From the $23 \mathrm{SNe}$ Ia at $z>1$ with HST data (Riess et al. 2004, 2007) we have learned: (1) that cosmic expansion was once decelerating before it recently began accelerating, (2) that dark energy, i.e., an energy density with $w<0$, was already present during this prior decelerating phase, (3) that SNe Ia at a look-back time of 10 Gyr appear both spectroscopically and photometrically similar to those seen locally, and (4) no rapid change is seen in $w(z)$ and thus no departure is yet seen from the cosmological constant, though the constraint on the time variation remains an order of magnitude worse than on the $w_{0}$.

SNe Ia play a central role, not only as distance indicators for cosmography, but also as major contributors to cosmic metal production and distribution. The measurement of high-redshift SN Ia rates is therefore integral to understanding the history of chemical enrichment. The high-redshift rate cannot easily be predicted from the star formation rate because the nature and, hence, the timescales of the process behind the growth of the white dwarf toward the Chandrasekhar mass are not known. The two leading, competing scenarios are accretion from a close binary companion-the single-degenerate scenario (Whelan \& Iben 1973; Nomoto 1982), or merger with another white dwarf, following loss of orbital energy and angular momentum by emission of gravitational waves - the double-
Table 2

Estimated Number of $z \geqslant 1$ SNe Ia to be Discovered During the CLASH Program

\begin{tabular}{lc}
\hline \hline $\begin{array}{l}\text { Redshift } \\
\text { Range }\end{array}$ & $\begin{array}{c}\text { Estimated Number } \\
\text { of SNe Ia Found }\end{array}$ \\
\hline $1.0 \leqslant z<1.5$ & $7-11$ \\
$1.5 \leqslant z<2.0$ & $4-13$ \\
$2.0 \leqslant z \leqslant 2.7$ & $0-4$ \\
$1.0 \leqslant z \leqslant 2.7$ & $11-28$ \\
\hline
\end{tabular}

Notes. These estimates are the $68 \%$ confidence intervals, accounting for both statistical and systematic uncertainties. The assumed SN Ia rate is from Graur et al. (2011).

degenerate scenario (Iben \& Tutukov 1984; Webbink 1984). One way to constrain the different progenitor scenarios is to measure the delay-time distribution (DTD) of SNe Ia. This is the distribution of times that elapse between a brief burst of star formation and the subsequent SN Ia explosions. Observations have suggested various different forms for the DTD (e.g., Dahlen et al. 2004, 2008; Mannucci et al. 2006; Pritchet et al. 2008). However, a number of more recent measurements and analyses point to a DTD that is a power law of index $\approx-1$ (Totani et al. 2008; Maoz et al. 2010, 2011; Maoz \& Badenes 2010; Brandt et al. 2010; Horiuchi \& Beacom 2010; Graur et al. 2011). Specifically, Graur et al. (2011) have shown that such a DTD fits well the measured SN Ia rate out to $z \approx 2$. Small sample sizes are the major limiting factor at high redshifts. Large high- $z \mathrm{SN}$ samples are therefore needed to resolve the issue, and control possible biases in cosmological studies (due to the evolving SN Ia channel mix).

The CLASH survey uses ACS in parallel with the cluster program to continue the discovery of SNe Ia at $1<z<1.5$, the objects which tell us about the variation in $w$. With WFC3 in parallel, CLASH will yield SNe Ia at $1.5<z<2.5$. Observations in this fully matter-dominated epoch provide the unique chance to test SN Ia distance measurements for the deleterious effects of evolution independent of our ignorance of dark energy (Riess \& Livio 2006). Because the SNe Ia are detected when these cameras are in parallel, they are far from the cluster core $(\sim 2 \mathrm{Mpc}$ at the median cluster redshift of $z=0.4$ ) and, hence, the effects of lensing are small (and correctable), making the SNe usable for improving the limits on the redshift variation of the dark energy equation of state. At $z<1$, SN Ia distance measurements are most sensitive to the static component of dark energy, $w_{0}$. At $1<z<1.5$, the measurements are most sensitive to the dynamic component, $w_{a}$. By $z>1.5$, the measurements are most sensitive to evolution if present (e.g., the changing $\mathrm{C} / \mathrm{O}$ ratio of the donor star), providing the means to diagnose and calibrate the degree of SN Ia evolution in dark energy measurements. Figure 4 shows how variations in the DE equation of state or an evolution in the white dwarf $\mathrm{C} / \mathrm{O}$ ratio can change the $\mathrm{SN}$ Ia distance modulus as a function of redshift.

Accounting for the systematic uncertainty introduced by the cosmic star formation history, Graur et al. (2011) predicted the SN Ia rate out to higher redshifts (their Figure 13), which we use here, in conjunction with the CLASH observational parameters, to estimate the number of $z \geqslant 1 \mathrm{SNe}$ Ia that will be discovered over the course of this program. These estimates are presented in Table 2 and are the $68 \%$ confidence intervals, accounting for both statistical and systematic uncertainties. 


\subsection{Detection and Characterization of $z>7$ Galaxies}

One of the most important goals in observational cosmology is to find the first generation of galaxies and understand their roles in the reionization of the intergalactic medium (IGM) between $z \sim 6$ and $z \sim 12$. Substantial progress has been made in recent years in finding objects at $z \sim 6-7$ (Bouwens et al. 2006, 2009; Bunker et al. 2010; Oesch et al. 2010a, 2010b; McLure et al. 2010; Capak et al. 2011), which are, at most, 700-900 Myr of age. We wish to find objects at even earlier epochs, in order to understand (1) how the first galaxies were formed through a hierarchical merging process; (2) how the chemical elements were generated and redistributed through the galaxies; (3) how the central black holes exerted influence over the galaxy formation; and (4) how these objects contributed to the end of the "dark ages."

The majority of galaxies at $z=6-7$ have been discovered via two methods: (1) deep pencil-beam surveys, such as the Hubble Ultra Deep Field (HUDF; Beckwith et al. 2006) and the Great Observatories Origins Deep Survey (Giavalisco et al. 2004) and (2) degree-size surveys with $10 \mathrm{~m}$ class groundbased telescopes, such as the Subaru Deep Field. Both use a color selection to search for "dropout" candidates-galaxies with deep IGM absorption at the wavelengths shortward of the redshifted Ly $\alpha$ break. These efforts have proven successful at finding objects at $z<7$, but there is growing evidence for a rapid decrease in the number of candidates at higher redshifts (Iye et al. 2006; Bouwens et al. 2006; Castellano et al. 2010). HST/WFC3 data yield $73 z \sim 7$ and $59 z \sim 8$ candidates (Bouwens et al. 2011). These results are based on $\sim 500$ orbits of HUDF observations. In comparison, $>600$ galaxies are found at $z \sim 6$ (Bouwens et al. 2007; Su et al. 2011). To date, there have been only two or three spectroscopically confirmed galaxies at $z>7$ (Lehnert et al. 2010; Ono et al. 2011; Schenker et al. 2011) and one spectrum for a gamma ray burst at $z \sim 8.2$ (Salvaterra et al. 2009; Tanvir et al. 2009). The reason, in part, is a lack of photons: at redshift $z=8$ and 10 , an $L^{*}$ galaxy would have an apparent magnitude in the first NIR detection band of 28.2 and 29.6 , respectively.

Gravitational lensing by clusters amplifies the flux of background sources considerably. These cosmic telescopes improve the efficiency of searches for relatively bright high-redshift galaxies (Bouwens et al. 2009; Maizy et al. 2010). In fact, the majority of $m<25.5 \mathrm{AB}, z \gtrsim 6.5$ galaxy candidates have been found in cluster fields (e.g., Kneib et al. 2004; Bradley et al. 2008; Zheng et al. 2009; Bradley et al. 2012; Schenker et al. 2011). Interestingly, most of these candidates are found in regions with amplification of $\sim 10$. Furthermore, lens models can be used to help discriminate between highly reddened objects and truly distant, high-redshift objects, as the projected positions of the lensed images are strong functions of the source redshifts.

Luminous $z>7$ galaxies are extremely valuable as their spectra can be used to determine the epoch of the IGM reionization. This is because only a tiny fraction of neutral hydrogen is needed to produce the high opacity of Ly $\alpha$ observed at $z \sim 6$. The damped $\operatorname{Ly} \alpha$ absorption profile that results from a completely neutral IGM (Miralda-Escude 1998) can be measured even at low-spectral resolution. Furthermore, direct measurements of the early star formation rate (via Ly $\alpha$ and $\mathrm{H} \alpha$ emission; Iye et al. 2006) can be derived from the spectra of bright high- $z$ galaxies. CLASH may detect dozens of relatively bright (magnified to $m<26.7 \mathrm{AB}$ ) $z>7$ galaxies, including some bright enough for spectroscopic follow-up. An

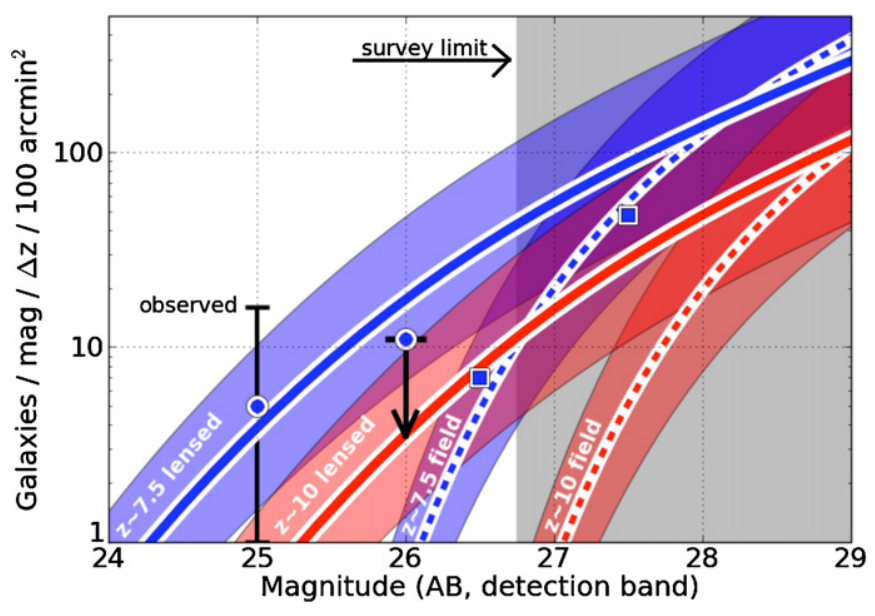

Figure 5. Estimated number counts in the total CLASH survey at $z \sim 7.5$ and 10 are plotted as the solid "lensed" blue and red lines, respectively, as functions of magnitudes in the detection bands F110W and F160W. Note the large uncertainties (shaded regions). An NIR survey of a comparable area $\left(100 \mathrm{arcmin}^{2}\right)$ in unlensed fields would have a $\sim 10 \times$ lower efficiency at these magnitudes (in agreement with Maizy et al. 2010). We have assumed an evolving Schechter luminosity function with $d M^{\star} / d z=0.36$ as derived by Bouwens et al. $(2006,2008)$ and plotted as the dashed lines with shaded uncertainties. Observed number counts from Bouwens et al. (2009) are plotted as squares. Lensing estimates were derived using the Zitrin et al. (2009) CL0024+17 mass model to simulate the magnifications of sources and reduction of source area. These estimates are in agreement with observed lensed counts in cluster fields plotted as circles (Bouwens et al. 2009).

(A color version of this figure is available in the online journal.)

NIR survey of a comparable area $\left(100 \operatorname{arcmin}^{2}\right)$ in unlensed fields would have a $\sim 10 \times$ lower efficiency. An estimate, albeit a highly uncertain one, of the CLASH high- $z$ detection efficiency enhancement is shown in Figure 5.

\subsection{Galaxy Evolution}

$\Lambda \mathrm{CDM}$ provides a robust theoretical framework for the evolution of DM halos. However, the formation of galaxies within these halos is governed by complicated baryonic interactions that are difficult to simulate on cosmological scales. The gap in our understanding of the connection between the formation of galaxies and that of their parent halos is illustrated by observations of galaxy "downsizing" (Cowie et al. 1996). $\Lambda$ CDM predicts that structures form hierarchically, with small halos forming early and later assembling into larger halos. In contrast, massive galaxies appear to have formed at early times, as evidenced by the redshift at which their star formation peaks (e.g., Cowie et al. 1996; Guzman et al. 1997; Brinchmann \& Ellis 2000; Juneau et al. 2005) and the old ages of their stellar populations at $z=0$ (e.g., Faber et al. 1995; Worthey 1996; Proctor \& Sansom 2002; Thomas et al. 2005). The stellar mass density of the universe is also increasing with cosmic time, as galaxies form stars, then shut down their star formation and, ultimately, accumulate on the red sequence (Bell et al. 2004; Faber et al. 2007).

Several open questions remain about the origin and nature of this stellar mass growth. Is it dominated by in situ star formation, or through mergers of existing stellar systems? How does the balance of these two processes change with galaxy mass and cosmic time? In a system dominated by hierarchical assembly, this is akin to asking whether most of the mass accreted by galaxies comes in as gas (e.g., the "cold flows" of Kereš et al. 2005; Dekel \& Birnboim 2006) or as stars (e.g., "dry mergers," 
Khochfar \& Burkert 2003; Bell et al. 2004). Theoretical expectations predict that the in situ population should be very centrally concentrated, while an accreted stellar component should extend to a larger radius (Oser et al. 2010). Thus, massive galaxies are predicted to have substantial gradients in the origin of their stars, with the innermost stars having formed in situ and the outer stars largely accreted through merging.

Recent work has demonstrated that massive, passive galaxies already exist at $z \sim 2$ (e.g., Trujillo et al. 2006; Kriek et al. 2009) and that these systems are much more compact than local massive galaxies (Trujillo et al. 2006; Longhetti et al. 2007; van Dokkum 2008), suggesting that they must grow significantly in size between $z \sim 2$ and $z \sim 0$. This process likely happens through "dry" (dissipationless) merging, since such massive galaxies are observed to have old stellar populations locally. There are also substantial populations of massive star-forming galaxies over a similar redshift range. These galaxies tend to exhibit large star-forming "clumps" (Cowie et al. 1995; van den Bergh 1996; Elmegreen et al. 2004a, 2004b), although at least some of these galaxies may form disks with coherent rotation (Genzel et al. 2011).

There are several observational challenges to measuring and characterizing these two modes of growth. It is difficult to resolve substructure in high-redshift galaxies, even from space. Both resolution and signal-to-noise $(\mathrm{S} / \mathrm{N})$ considerations limit observations to the upper end of the mass function. Searches that use a small number of imaging filters struggle to identify the redshifts (and therefore the masses) of the targets and can only poorly constrain their spectral energy distributions (SEDs) and stellar populations. Studies that rely on emission lines probe only the assembly of star-forming galaxies.

The CLASH program is well suited for studying galaxy assembly at $z<3$. The high magnification and spatial stretching of strongly lensed distant galaxies, coupled with the broad 16band photometry and resulting photometric redshifts, enables the measurement of star formation rates and stellar ages in each lensed galaxy over many resolution elements. The magnification makes it possible to resolve substructures in high-redshift lensed galaxies further down the galaxy mass function than is possible in unlensed galaxies at similar redshifts. In addition, with CLASH, we can contrast the relative properties of galaxy cores versus their outer regions, and (where they exist) the properties of substructures such as clumps, spiral arms, and ongoing mergers. In the full CLASH sample of 25 clusters, we expect to find several strongly lensed background galaxies per cluster that are suitable for such analyses, providing a sample of 50-100 galaxies with $1<z<3$.

\section{CLASH CLUSTER SAMPLE}

The CLASH program is robustly measuring galaxy cluster DM profiles and concentrations for a systematic comparison with those realized in cosmological simulations. Specifically, our cluster sample size and selection criteria were chosen to allow the robust measurement of deviations from the predicted cluster concentration distribution of $\sim 15 \%$ or more at high statistical confidence ( $\sim 99 \%$ C.L.) given a relatively unbiased ensemble of clusters (Section 2.1).

\subsection{Cluster Sample Selection}

To date, robust joint SL+WL analyses have only been performed on a small, highly biased sample of five to ten clusters (Table 1 and Figure 3). These clusters were selected for study primarily based on their gravitational lensing strength, which tends to preferentially select systems with higher central matter concentrations. To establish a sample that is largely free of lensing bias, we selected 20 massive clusters from X-ray-based compilations of dynamically relaxed systems. Sixteen of these 20 clusters were taken from the Allen et al. (2008) compilation of massive relaxed clusters. Clusters in our X-ray-selected subsample all have $T_{x} \geqslant 5 \mathrm{keV}$ and exhibit a high degree of dynamical relaxation as evidenced by Chandra X-ray Observatory images that show well-defined central surface brightness peaks and nearly concentric isophotes. The clusters, in general, also show minimal evidence for departures from hydrostatic equilibrium in X-ray pressure maps. Most of the 20 clusters have smooth and only mildly elliptical $(\langle\epsilon\rangle=0.19) \mathrm{X}$-ray emission and a BCG within a projected distance of $23 \mathrm{kpc}$ of the X-ray centroid. No lensing information was used a priori to select them in order to ensure that they are not preferentially aligned along the line of sight, in contrast with a purely lensing-selected sample, where the surface mass density is, on average, biased upward along the line of sight by intrinsic triaxiality (Hennawi et al. 2007; Oguri et al. 2009; Meneghetti et al. 2010, 2011). A handful of the clusters in the CLASH X-ray-selected subset have some evidence for departures from symmetric X-ray surface brightness distributions. These systems are briefly discussed in Section 3.3.

These 20 clusters comprise the primary sample for our DM distribution studies. Existing X-ray data will be used to measure the gas density profile for subtraction from the total mass profile (as applied by Lemze et al. 2008 for A1689), which is necessary when making detailed comparison with the predictions of DMonly simulations. Although these clusters are X-ray selected, one or more lensed arcs are indeed visible in all of the clusters for which sufficiently high-quality imaging was available. This indicates that the X-ray-selected clusters in our sample have Einstein radii in the range $15^{\prime \prime}-35^{\prime \prime}$. X-ray images from the $\mathrm{ACCEPT}^{25}$ database for these $20 \mathrm{CLASH}$ clusters are shown in Figure 6.

In addition, CLASH includes five clusters selected based solely on their exceptional strength as gravitational lenses (large Einstein radii, $\theta_{\text {Ein }}>35^{\prime \prime}$ for $z_{s}=2$ ). Analysis of these clusters will allow us to further quantify the lensing selection bias toward high concentrations. The excellent strong-lensing data yielded by these clusters will enable derivation of some of the highest resolution DM maps which may be obtained (as in Coe et al. 2010). The primary motivation for selecting these five "high-magnification" clusters, however, was to significantly increase the likelihood of discovering very highly magnified high-redshift galaxies (Section 2.3). While most of the CLASH clusters lie behind regions of low Galactic extinction (median $E(B-V)=0.026)$, there is one cluster, MACS2129.4-0741, with significant amounts of Galactic cirrus located northeast of the cluster core. Because this cluster has one of the larger Einstein radii known, its inclusion in the "high-magnification" sample was deemed worthwhile in spite of the presence of this cirrus.

Figure 7 shows, respectively, the distribution of the projected separation between the BCG and the peak of the X-ray surface brightness and the distribution of the ellipticity of the X-ray emission for the CLASH sample. Ellipticity measurements are from Maughan et al. (2008). The X-ray-selected cluster

\footnotetext{
25 ACCEPT $=$ Archive of Chandra Cluster Entropy Profile Tables. See http://www.pa.msu.edu/astro/MC2/accept/ for more information
} 


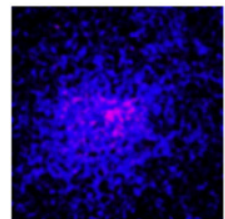

Abell 209

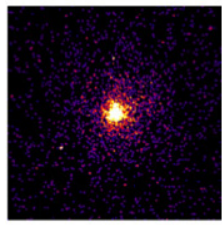

MACS 0329-0211

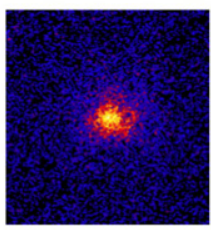

Cป1226+3332

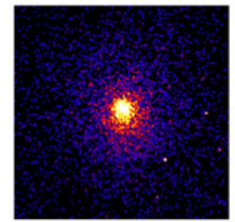

MACS $1720+3536$

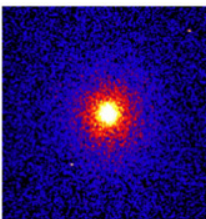

Abell 383

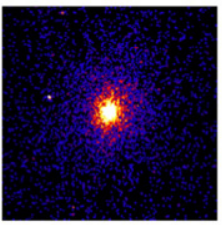

MACS 0429-0253

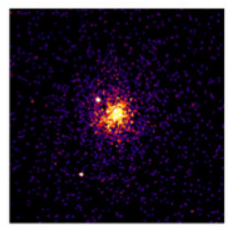

MACS $1311-0310$

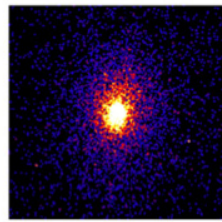

MACS 1931-2634

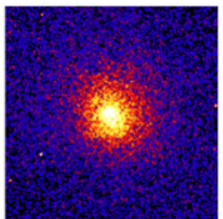

Abell 611

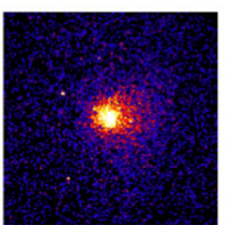

MACS $0744+3927$

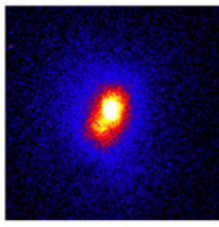

RXJ 1347-1145

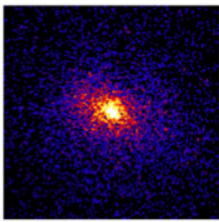

RXJ 2129+0005

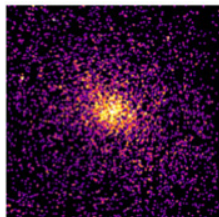

Abell 1423

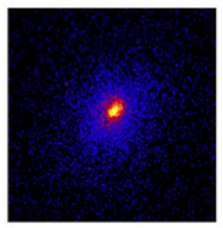

MACS $1115+0129$

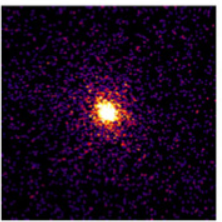

MACS $1423+2404$

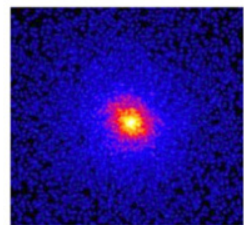

MS-2137

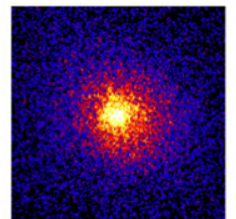

Abell 2261

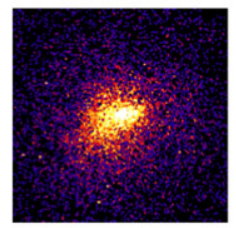

MACS 1206-0847

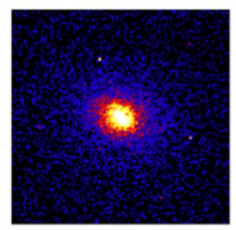

RXJ $1532+3020$

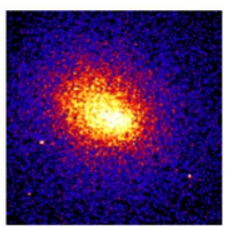

RXJ 2248-4431

Figure 6. Cutouts of Chandra X-ray images centered on the $20 \mathrm{CLASH}$ clusters in the X-ray-selected subsample. These images are taken from the Archive of Chandra Cluster Entropy Profile Tables (ACCEPT). Each cutout subtends 3.45 arcmin, which is nearly the same as the ACS/WFC field of view on HST.

(A color version of this figure is available in the online journal.)
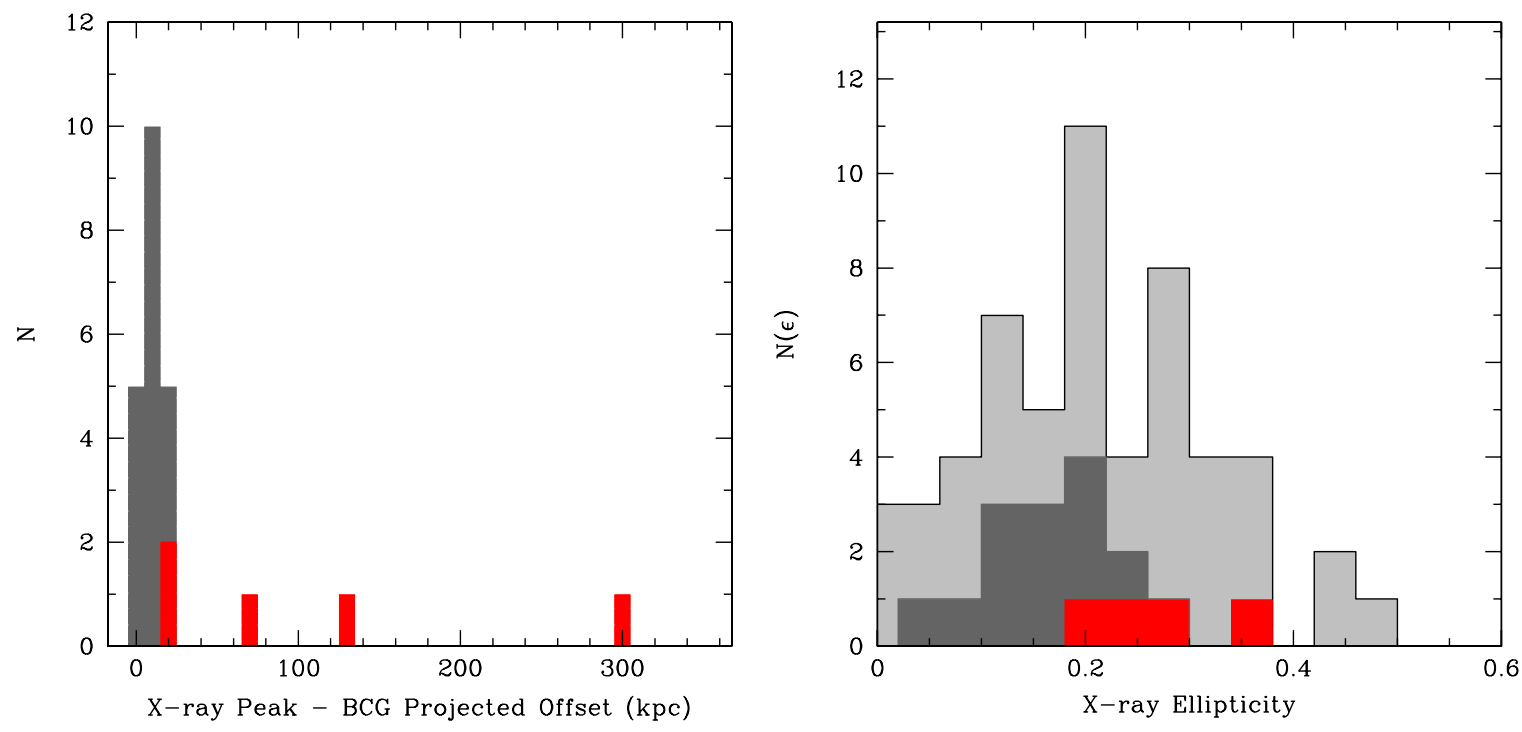

Figure 7. Left: the distribution of the projected separation between the brightest cluster galaxy (BCG) and the peak of the X-ray surface brightness for the $\mathrm{X}$-ray-selected (dark gray) and Einstein-radii selected (red) cluster subsamples. The average projected BCG-X-ray peak separation for the X-ray-selected sample is 10.4 kpc. Right: the distribution of the ellipticity of the X-ray emitting intracluster gas for the X-ray-selected (dark gray) and high-magnification-selected (red) cluster subsamples. The distribution of the ellipticities of the 96 clusters in the Maughan et al. (2008) sample that are not in common with CLASH is shown in the light gray histogram. The mean X-ray emission ellipticities for the X-ray and Einstein-radii selected cluster samples are 0.19 and 0.28 , respectively.

(A color version of this figure is available in the online journal.)

subsample exhibits, on average, a very small offset between the BCG and peak X-ray flux. The X-ray-selected cluster subsample also exhibits a lower average ellipticity than our highmagnification (Einstein-radii selected) sample but is consistent with the mean ellipticity of the larger Maughan et al. (2008) sample.

The 25 clusters in the CLASH program are presented in Table 3 and some of their key X-ray properties are given 
Table 3

The CLASH Cluster Sample and HST Observing Plan

\begin{tabular}{|c|c|c|c|c|c|c|c|}
\hline Cluster & $\alpha_{\mathrm{J} 2000}$ & $\delta_{\mathrm{J} 2000}$ & $z_{\text {Clus }}$ & $\begin{array}{l}\text { HST } \\
\text { Cycle }\end{array}$ & $\begin{array}{c}\text { CLASH } \\
\text { Orbits }\end{array}$ & $\begin{array}{c}\text { Program } \\
\text { ID }\end{array}$ & $\begin{array}{l}\text { Archival } \\
\text { Orbits }^{c}\end{array}$ \\
\hline \multicolumn{8}{|l|}{ X-ray Selected Clusters: } \\
\hline Abell 209 & $01: 31: 52.57$ & $-13: 36: 38.8$ & 0.206 & 19 & 20 & 12451 & (3) \\
\hline Abell 383 & $02: 48: 03.36$ & $-03: 31: 44.7$ & 0.187 & 18 & 20 & 12065 & (3) \\
\hline MACS0329.7-0211 & $03: 29: 41.68$ & $-02: 11: 47.7$ & 0.450 & 19 & 20 & 12452 & $(2.5)$ \\
\hline MACS0429.6-0253 & $04: 29: 36.10$ & $-02: 53: 08.0$ & 0.399 & 20 & 20 & 12788 & $(0.5)$ \\
\hline MACS0744.9+3927 & $07: 44: 52.80$ & $+39: 27: 24.4$ & 0.686 & 18 & 17 & 12067 & 6 \\
\hline Abell 611 & 08:00:56.83 & $+36: 03: 24.1$ & 0.288 & 19 & 18 & 12460 & 2 \\
\hline MACS1115.9+0129 & $11: 15: 52.05$ & $+01: 29: 56.6$ & 0.352 & 19 & 20 & 12453 & 0.5 \\
\hline Abell 1423 & $11: 57: 17.26$ & $+33: 36: 37.4$ & 0.213 & 20 & 20 & 12787 & $(0.5)$ \\
\hline MACS1206.2-0847 & $12: 06: 12.28$ & $-08: 48: 02.4$ & 0.440 & 18 & 20 & 12069 & $0.5+(0.5)$ \\
\hline CLJ1226.9+3332 & $12: 26: 58.37$ & $+33: 32: 47.4$ & 0.890 & 20 & 18 & 12791 & 16 \\
\hline MACS1311.0-0310 & 13:11:01.67 & $-03: 10: 39.5$ & 0.494 & 20 & 20 & 12789 & 0 \\
\hline RXJ1347.5-1145 & $13: 47: 30.59$ & $-11: 45: 10.1$ & 0.451 & 18 & 15 & 12104 & $6+(0.5)$ \\
\hline MACS1423.8+2404 & $14: 23: 47.76$ & $+24: 04: 40.5$ & 0.545 & 20 & 17 & 12790 & 5 \\
\hline RXJ1532.9+3021 & $15: 32: 53.78$ & $+30: 20: 58.7$ & 0.345 & 19 & 20 & 12454 & $(0.5)$ \\
\hline MACS1720.3+3536 & $17: 20: 16.95$ & $+35: 36: 23.6$ & 0.391 & 19 & 20 & 12455 & $(0.5)$ \\
\hline Abell 2261 & $17: 22: 27.25$ & $+32: 07: 58.6$ & 0.224 & 18 & 20 & 12066 & $(0.5)$ \\
\hline MACS1931.8-2635 & $19: 31: 49.66$ & $-26: 34: 34.0$ & 0.352 & 19 & 20 & 12456 & 0 \\
\hline RXJ2129.7+0005 & $21: 29: 39.94$ & $+00: 05: 18.8$ & 0.234 & 19 & 20 & 12457 & (1) \\
\hline MS2137-2353 & $21: 40: 15.18$ & $-23: 39: 40.7$ & 0.313 & 18 & 18 & 12102 & $5+(7)$ \\
\hline RXJ2248.7-4431 (Abell 1063S) & $22: 48: 44.29$ & $-44: 31: 48.4$ & 0.348 & 19 & 20 & 12458 & $(0.5)$ \\
\hline \multicolumn{8}{|l|}{ High Magnification Clusters: } \\
\hline MACS0416.1-2403 & 04:16:09.39 & $-24: 04: 03.9$ & $0.42^{\mathrm{b}}$ & 19 & 20 & 12459 & (1) \\
\hline MACS0647.8+7015 & $06: 47: 50.03$ & $+70: 14: 49.7$ & 0.584 & 18 & 18 & 12101 & 9 \\
\hline MACS0717.5+3745 & $07: 17: 31.65$ & $+37: 45: 18.5$ & 0.548 & 18 & 17 & 12103 & 7 \\
\hline MACS1149.6+2223 & $11: 49: 35.86$ & $+22: 23: 55.0$ & 0.544 & 18 & 18 & 12068 & 5 \\
\hline MACS2129.4-0741 & $21: 29: 26.06^{\mathrm{a}}$ & $-07: 41: 28.8^{\mathrm{a}}$ & 0.570 & 18 & 18 & 12100 & 5 \\
\hline
\end{tabular}

Notes.

${ }^{\text {a }}$ Central cluster coordinates derived from optical image instead of X-ray image.

${ }^{\mathrm{b}}$ Cluster redshift for MACS0416.1-2403 is based on Chandra X-ray spectrum (this work). Uncertainty on this value is \pm 0.02 . For this fit, the core was not excluded. To maximize the counts, an aperture of $714 \mathrm{kpc}$ was used, binned to achieve a minimum of 20 counts per energy bin. The background in the $0.7-7.0 \mathrm{keV}$ range was fitted from the Chandra deep fields.

c Archival ACS or WFPC2 imaging data only; WFPC2 orbits shown in parentheses. Archival ACS images, when available, are used in conjunction with new CLASH data to achieve the desired depths in all filters.

in Table 4, including the bolometric luminosity (defined for convenience to be between 0.1 and $100 \mathrm{keV}$ ), temperature, and cluster-to-solar $[\mathrm{Fe} / \mathrm{H}]$ ratio, where the solar abundance reference is from Anders \& Grevesse (1989). Table 4 also lists the source of the X-ray selection in Column 6. The CLASH sample is drawn heavily from the Abell and MACS cluster catalogs (Abell 1958; Abell et al. 1989; Ebeling et al. 2001, 2007, 2010). The CLASH clusters span almost an order of magnitude in mass $\left(\sim 5\right.$ to $\left.\sim 30 \times 10^{14} M_{\odot}\right)$. These clusters were also selected to cover a wide redshift range $(0.18<z<0.90$ with a median $z_{\text {med }}=0.40$ ) allowing us to probe the full $c(M, z)$ relations expected from simulations.

The X-ray parameters in Table 4 are derived by us using CIAO v4.3 and CALDB v4.4.3. We filtered the Chandra data for flares and reprojected the deep Chandra background fields to match the cluster observations. The background data were also filtered for "status $=0$ " events to be suitable for use with the VFAINT mode data. To obtain X-ray luminosities and temperatures uniformly across the sample, we extracted spectra from apertures with radii of $714 \mathrm{kpc}\left(500 h_{100}^{-1} \mathrm{kpc}\right)$ and excluded the central $71.4 \mathrm{kpc}\left(50 h_{100}^{-1} \mathrm{kpc}\right)$. These "coreexcised" spectra provide X-ray temperature estimates that are relatively unaffected by the presence or absence of a cool core (e.g., Markevitch 1998). Figure 8 shows the X-ray temperature, $T_{x}$, as a function of the scaled bolometric luminosity, $L_{x} / E(z)$, for CLASH clusters using the data in Table 4. The X-ray luminosities are scaled assuming self-similar evolution, $E(z)=$ $\sqrt{\Omega_{m}(1+z)^{3}+\Lambda}$, to allow direct comparison to the low-redshift luminosity temperature relationships from Markevitch (1998) and Pratt et al. (2009) ( $L_{2}$ (measured within 0.15-1.00 $R_{500}$ ) versus $T_{3}\left(0.15-0.75 R_{500}\right)$, to be specific). We verified that the deep background particle event rates matched that seen in the cluster spectra between 9 and $12 \mathrm{keV}$. The X-ray counts were binned to a minimum of 20 counts per energy bin. Since the extracted spectra were dominated by source counts, the results were not very sensitive to the background scaling. To estimate the cluster temperature, we used XSPEC 12.6.0q to fit the $\mathrm{X}$-ray spectra between 0.7 and $7.0 \mathrm{keV}$. We assumed a single temperature plasma model (the XSPEC apec model) absorbed by a Galactic hydrogen column fixed to the value obtained from the Bell survey (Dickey \& Lockman 1990). The metallicity, temperature, and normalization were allowed to be free. The best-fitted temperatures and $1 \sigma$ error bars, and the bolometric $\mathrm{X}$-ray luminosities are reported in Table 4.

\subsection{Cluster Sample Size Requirements}

The required size of our "relaxed" cluster sample is derived from the goal to measure "average" cluster concentrations to $\sim 10 \%$ (after accounting for variations in mass and redshift) and 
Table 4

X-ray Properties of the CLASH Cluster Sample

\begin{tabular}{|c|c|c|c|c|c|c|}
\hline Cluster & $\begin{array}{c}\mathrm{kT} \\
(\mathrm{keV})\end{array}$ & $\begin{array}{c}\left(\mathrm{L}_{B o l}\right)^{\mathrm{a}} \\
\left(10^{44} \mathrm{erg} \mathrm{s}^{-1}\right)\end{array}$ & $\begin{array}{c}{[\mathrm{Fe} / \mathrm{H}] \text { Ratio }} \\
{\text { (Cluster/Solar })^{\mathrm{c}}}\end{array}$ & $\begin{array}{c}\text { Ellipticity } \\
\epsilon\end{array}$ & $\begin{array}{l}\text { Centroid Shift }{ }^{\mathrm{b}} \\
\langle w\rangle\left(10^{-2} R_{500}\right)\end{array}$ & $\begin{array}{l}\text { X-ray Morphology } \\
\text { Reference }\end{array}$ \\
\hline \multicolumn{7}{|l|}{ X-ray Selected Clusters: } \\
\hline Abell 209 & $7.3 \pm 0.54$ & $12.7 \pm 0.3$ & $0.18 \pm 0.09$ & $0.21 \pm 0.01$ & $0.55 \pm 0.05$ & 1 \\
\hline Abell 383 & $6.5 \pm 0.24$ & $6.7 \pm 0.2$ & $0.59 \pm 0.10$ & $0.04 \pm 0.01$ & $0.18 \pm 0.02$ & 2 \\
\hline MACS0329.7-0211 & $8.0 \pm 0.50$ & $17.0 \pm 0.6$ & $0.48 \pm 0.10$ & $0.15 \pm 0.03$ & $1.38 \pm 0.13$ & 2,3 \\
\hline MACS0429.6-0253 & $6.0 \pm 0.44$ & $11.2 \pm 0.5$ & $0.34 \pm 0.11$ & $0.21 \pm 0.02$ & $0.38 \pm 0.03$ & 2,3 \\
\hline MACS0744.9+3927 & $8.9 \pm 0.80$ & $29.1 \pm 1.2$ & $0.37 \pm 0.10$ & $0.11 \pm 0.02$ & $1.41 \pm 0.13$ & 2,3 \\
\hline Abell 611 & $7.9 \pm 0.35$ & $11.7 \pm 0.2$ & $0.30 \pm 0.07$ & $\ldots$ & $\ldots$ & 2 \\
\hline MACS1115.9+0129 & $8.0 \pm 0.40$ & $21.1 \pm 0.4$ & $0.32 \pm 0.06$ & $\cdots$ & $\cdots$ & 2,3 \\
\hline Abell 1423 & $7.1 \pm 0.65$ & $7.8 \pm 0.2$ & $0.23 \pm 0.13$ & $\cdots$ & $\cdots$ & 4 \\
\hline MACS1206.2-0847 & $10.8 \pm 0.60$ & $43.0 \pm 1.0$ & $0.25 \pm 0.09$ & $\ldots$ & $\ldots$ & 3,4 \\
\hline CLJ1226.9+3332 & $13.8 \pm 2.80$ & $34.4 \pm 3.0$ & $0.36 \pm 0.25$ & $0.10 \pm 0.03$ & $1.30 \pm 0.11$ & 2 \\
\hline MACS1311.0-0310 & $5.9 \pm 0.40$ & $9.4 \pm 0.4$ & $0.42 \pm 0.10$ & $0.08 \pm 0.02$ & $0.39 \pm 0.03$ & 2,3 \\
\hline RXJ1347.5-1145 & $15.5 \pm 0.60$ & $90.8 \pm 1.0$ & $0.20 \pm 0.06$ & $0.26 \pm 0.01$ & $0.63 \pm 0.05$ & 2 \\
\hline MACS1423.8+2404 & $6.5 \pm 0.24$ & $14.5 \pm 0.4$ & $0.35 \pm 0.06$ & $0.17 \pm 0.03$ & $0.25 \pm 0.02$ & 2,3 \\
\hline RXJ1532.9+3021 & $5.5 \pm 0.40$ & $20.5 \pm 0.9$ & $0.52 \pm 0.12$ & $0.18 \pm 0.02$ & $0.07 \pm 0.01$ & 2 \\
\hline MACS1720.3+3536 & $6.6 \pm 0.40$ & $13.3 \pm 0.5$ & $0.29 \pm 0.09$ & $0.17 \pm 0.02$ & $0.57 \pm 0.05$ & 2,3 \\
\hline Abell 2261 & $7.6 \pm 0.30$ & $18.0 \pm 0.2$ & $0.31 \pm 0.06$ & $0.10 \pm 0.01$ & $0.71 \pm 0.06$ & 5 \\
\hline MACS1931.8-2635 & $6.7 \pm 0.40$ & $20.9 \pm 0.6$ & $0.26 \pm 0.08$ & $0.30 \pm 0.01$ & $0.28 \pm 0.02$ & 2,3 \\
\hline RXJ2129.7+0005 & $5.8 \pm 0.40$ & $11.4 \pm 2.0$ & $0.33 \pm 0.10$ & $0.26 \pm 0.02$ & $0.55 \pm 0.05$ & 2 \\
\hline MS2137-2353 & $5.9 \pm 0.30$ & $9.9 \pm 0.3$ & $0.41 \pm 0.08$ & $\ldots$ & $\ldots$ & 2 \\
\hline RXJ2248.7-4431 (Abell 1063S) & $12.4 \pm 0.60$ & $69.5 \pm 0.1$ & $0.39 \pm 0.06$ & $0.20 \pm 0.01$ & $0.74 \pm 0.06$ & 6 \\
\hline \multicolumn{7}{|l|}{ High Magnification Clusters: } \\
\hline MACS0416.1-2403 & $7.5 \pm 0.80$ & $16.0 \pm 0.9$ & $0.40 \pm 0.14$ & $\cdots$ & $\cdots$ & 3 \\
\hline MACS0647.8+7015 & $13.3 \pm 1.80$ & $32.5 \pm 2.1$ & $0.30 \pm 0.19$ & $0.36 \pm 0.02$ & $0.62 \pm 0.06$ & 3 \\
\hline MACS0717.5+3745 & $12.5 \pm 0.70$ & $55.8 \pm 1.1$ & $0.22 \pm 0.07$ & $0.30 \pm 0.01$ & $2.11 \pm 0.18$ & 3 \\
\hline MACS1149.6+2223 & $8.7 \pm 0.90$ & $30.2 \pm 1.2$ & $0.24 \pm 0.11$ & $0.25 \pm 0.02$ & $1.21 \pm 0.10$ & 3 \\
\hline MACS2129.4-0741 & $9.0 \pm 1.20$ & $22.6 \pm 1.5$ & $0.40 \pm 0.17$ & $0.19 \pm 0.03$ & $1.56 \pm 0.14$ & 3 \\
\hline
\end{tabular}

Notes.

a The X-ray bolometric luminosity covers the energy range $0.1-100 \mathrm{keV}$.

b The X-ray ellipticity, $\epsilon$, and centroid shift, $\langle w\rangle$, values are from Maughan et al. (2008).

c The solar abundance reference values used here are from Anders \& Grevesse (1989).

References. (1) Maughan et al. (2008); (2) Allen et al. (2008); (3) Ebeling et al. (2007); (4) Cavagnolo et al. (2008); (5) Mantz et al. (2010); (6) CLASH team selected cluster.

to detect a $\sim 15 \%$ deviation from the concentrations of simulated clusters at $99 \%$ confidence.

Statistically, if we assume measurement errors are independent and normally distributed, then the number of clusters, $N_{\text {clus }}$, needed to measure the average concentration to a fractional accuracy of $f$ is just

$$
N_{\text {clus }}=\left(\sigma_{\text {tot }} / f\right)^{2}
$$

where the total scatter in an individual measurement of the concentration, $\sigma_{\text {tot }}$, is

$$
\sigma_{\text {tot }}^{2}=\sigma_{\text {int }}^{2}+\sigma_{\text {LSS }}^{2}+\sigma_{\text {meas }}^{2}
$$

and includes contributions from intrinsic variation (including the effects of orientation averaging of triaxial halo shapes), variations due to intervening large-scale structure (LSS), and measurement uncertainties. Concentrations of relaxed simulated cluster halos have intrinsic scatters of $\sim 30 \%$ (e.g., Neto et al. 2007; Macciò et al. 2008; Duffy et al. 2008). LSS can scatter concentration measurements by $\sim 13 \%$ (Hoekstra et al. 2002). And we conservatively estimate measurement uncertainties to be $\sim 37 \%$ as follows based on empirical data (though analysis of simulated data suggests $\sim 11 \%$ accuracy is possible; Meneghetti et al. 2010).

Analysis of CL0024+17 yielded a concentration measurement uncertainty of $\sim 22 \%$ (Umetsu et al. 2010), which we will use here as a baseline. We assume here that our precisions will be dominated by the quality of our SL data and that our measurement uncertainty will scale roughly as $\sigma \propto 1 / \sqrt{N_{\operatorname{arc}}} \propto 1 / R_{E}$, where for CL0024+17 with $R_{E} \sim 35^{\prime \prime}, N_{\text {arc }}=33$ arcs were detected. Note that we have also assumed $N_{\text {arc }} \propto R_{E}^{2}$. Based on archival WFPC2 and ACS images, we measured a mean $R_{E} \approx 20^{\prime \prime}$ for our sample of 20 relaxed clusters, which should conservatively yield $N_{\text {arc }} \sim 12$ arcs per cluster. This scales the $\sim 22 \%$ uncertainty for CL0024+17 with 33 arcs up to a conservative $\sim 37 \%$ for an average cluster with just 12 arcs.

Adopting $\sigma_{\text {int }}=0.30, \sigma_{\text {LSS }}=0.13$, and $\sigma_{\text {meas }}=0.37$, we find $\sigma_{\text {tot }}=0.49$, and hence, if $f=0.10$ then $N_{\text {clus }} \sim 24$. This empirical sample size estimate is consistent with one derived from numerical simulations of strong lensing, as shown in Figure 9. The approximately log-normal distribution of DM halo concentrations seen in such simulations (Meneghetti et al. $2010)$ indicates that $\sim 20$ massive clusters $\left(M_{\text {vir }}>5 \times 10^{14} M_{\odot}\right)$ are required to detect $15 \%$ deviations from expectations in the standard cosmological model at $99 \%$ confidence.

Note that we may expect deviations to be greater than $15 \%$ if results from lensing-selected clusters are any indication. Simulated $10^{15} M_{\odot} h^{-1}$ clusters at $z=0$ typically have $c_{\text {vir }} \sim 5$ (e.g., Duffy et al. 2008). For this fiducial cluster, Oguri et al. (2009) instead find $c_{\text {vir }} \sim 12$ for observed clusters (based on a fit to values observed for 10 lensing clusters accounting for the various masses and redshifts). Even after including a 50\% lensing bias, these observed concentrations are still 


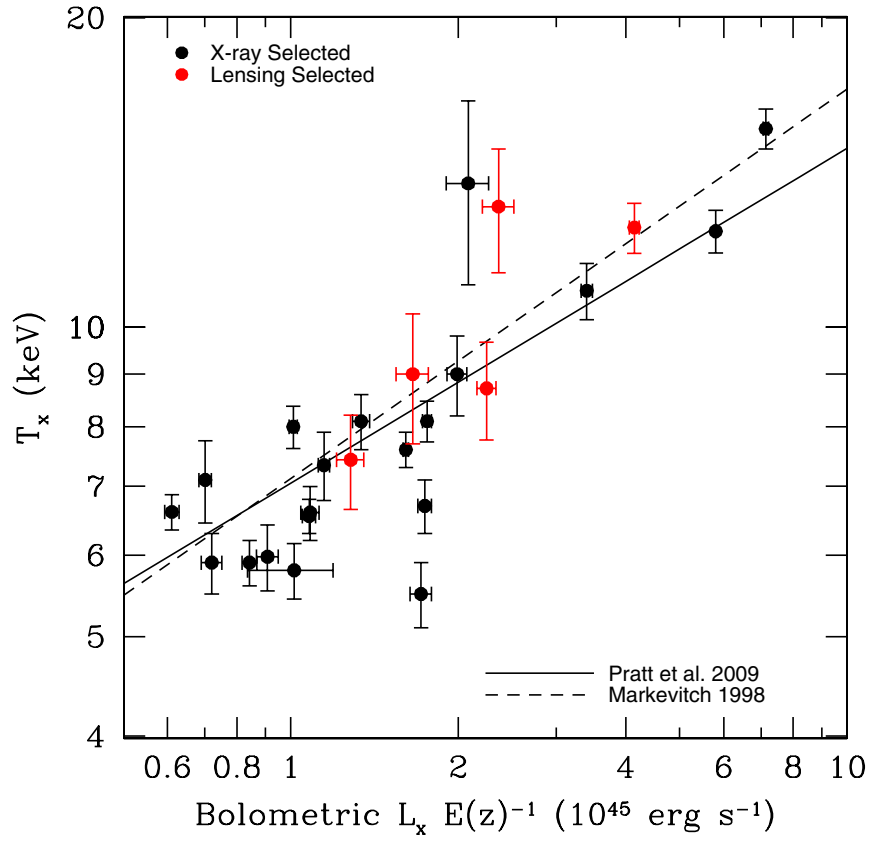

Figure 8. X-ray temperature, $T_{x}$, as a function of the scaled bolometric luminosity, $L_{x} / E(z)$, for the CLASH cluster sample. Data points are from Table 4. The X-ray luminosities are scaled assuming self-similar evolution to allow direct comparison to the low-redshift luminosity temperature relationships from Markevitch (1998) and Pratt et al. (2009). The $L_{x}-T_{x}$ relations from Markevitch (1998) and Pratt et al. (2009) $\left(L_{2}\right.$ (measured within 0.15-1.00 $\left.R_{500}\right)$ vs. $\left.T_{3}\left(0.15-0.75 R_{500}\right)\right)$ are shown. CLASH clusters are representative of these larger X-ray cluster samples.

(A color version of this figure is available in the online journal.)

$\sim 70 \%$ greater than expectations. Being more conservative and assuming a factor of two (100\%) lensing bias (Meneghetti et al. 2010), the observed concentrations would still be $\sim 20 \%$ greater than expectations. Although as discussed in Section 2.1, more recent simulations (Prada et al. 2011) may alleviate these discrepancies.

\subsection{Notes on Clusters with Possible Substructure}

While our X-ray selection criteria favor the inclusion of highly relaxed clusters in the CLASH sample, for eight of our clusters the dynamical state is somewhat ambiguous. Some researchers have reported evidence for substructure in the X-ray surface brightness profiles of these eight clusters. The presence of substructure may suggest that a cluster is not fully dynamically relaxed. We summarize this evidence below. We note that the presence of a minor degree of substructure does not inhibit our ability to determine a cluster's mass profile characteristics. In simulations, relaxed clusters have DM concentrations that are, on average, $\sim 20 \%$ higher than the general cluster population (Duffy et al. 2008; Prada et al. 2011).

1. A209. This system is marginally unrelaxed according to Smith et al. (2005). They based their relaxed/unrelaxed characterization mainly on a mass ratio between the cluster main component and the total mass (both at $r<500 \mathrm{kpc}$ ). Clusters with mass ratio values below 0.95 are considered unrelaxed. Their value for A209 is $0.87 \pm 0.06$. Maughan et al. (2008, hereafter M08) derived an ellipticity of $\epsilon=$ $0.21 \pm 0.01$ for this cluster. Gilmour et al. (2009, hereafter G09) find it to be relaxed based on a visual examination of its X-ray morphology.

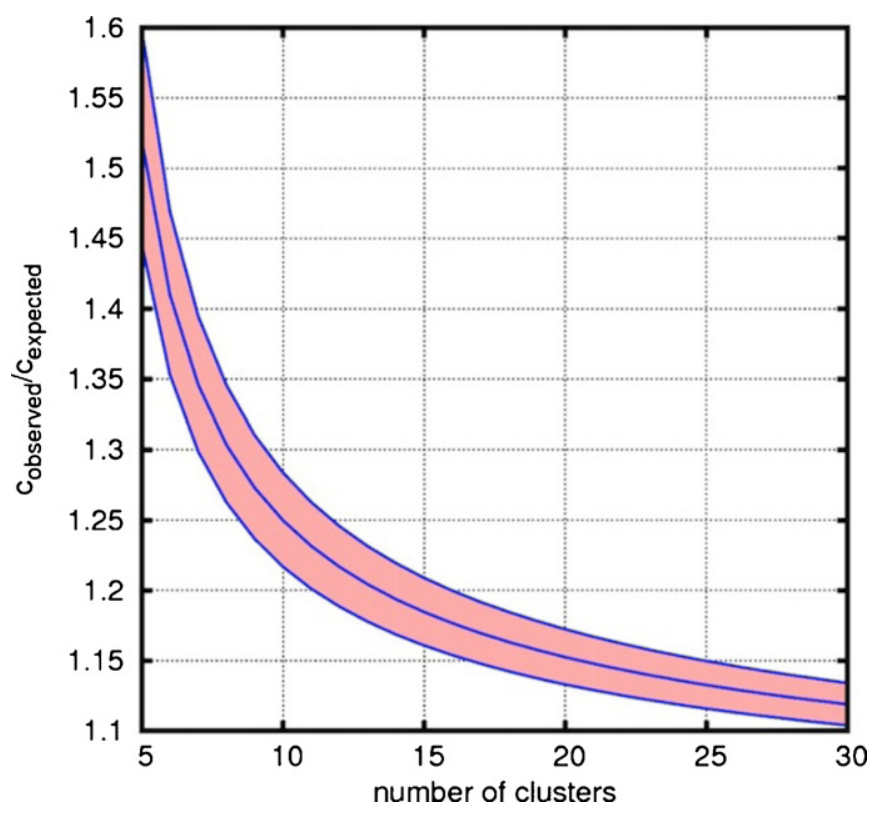

Figure 9. Mean "overconcentration" ratio necessary to reject with $99 \%$ confidence the hypothesis that measured concentrations are drawn from a sample with the expected values. Average deviations of $\sim 15 \%$ from expectations will be detected with $99 \%$ confidence based on our relaxed sample of 20 clusters. We have adopted the finding from $\Lambda \mathrm{CDM}$ simulations that DM halo concentrations are log-normally distributed with a standard deviation of $\sigma \approx 0.25 \pm 0.03$ (e.g., Duffy et al. 2008). The shaded band includes this uncertainty.

(A color version of this figure is available in the online journal.)

2. MACSJ0329.7-0211 and CLJ1226.9+3332. These two clusters are included in the A08 compilation (as well as in Schmidt \& Allen 2007, hereafter SA07), and were classified in these works as dynamically relaxed. However, M08 report that both exhibit evidence for substructure.

3. MACSJ0744.9+3927. This cluster shows evidence for substructure (SA07; M08). On the other hand, G09 find it to be relaxed based on a visual examination of its X-ray morphology.

4. MACSJ1206.2-0848. In both optical and X-ray images the cluster appears close to relaxed in projection, with a pronounced X-ray peak at the location of the BCG. However, some evidence of merger activity along the line of sight may be suggested by the very high velocity dispersion of $1580 \mathrm{~km} \mathrm{~s}^{-1}$. G09 visually classify this cluster as relaxed.

5. RXJ1347.5-1145. Significant substructure is observed in this system (SA07; M08). M08 measure an average ellipticity of the X-ray isophotes of $\epsilon=0.26 \pm 0.01$.

6. A2261. M08 found a small level of substructure and G09 classified it as disturbed.

7. RXJ2248.7-4431. M08 found this cluster to be slightly elliptical $\epsilon=0.2 \pm 0.01$ and with some level of substructure, while G09 found it to be relaxed.

\section{SURVEY DESIGN AND IMPLEMENTATION}

The CLASH program consists of 524 HST orbits, including 50 for SN follow-up. The bulk of the program (474 orbits) will be used to image 25 galaxy clusters, each to a depth of 20 orbits divided among 16 HST/ACS and WFC3 filters as shown in Table 5 and Figure 10. The number of required cluster orbits is reduced from 500 to 474, as some of these filter depths have already been achieved in existing data. Columns $6-8$ in Table 3 provide the number of new orbits allocated, the 


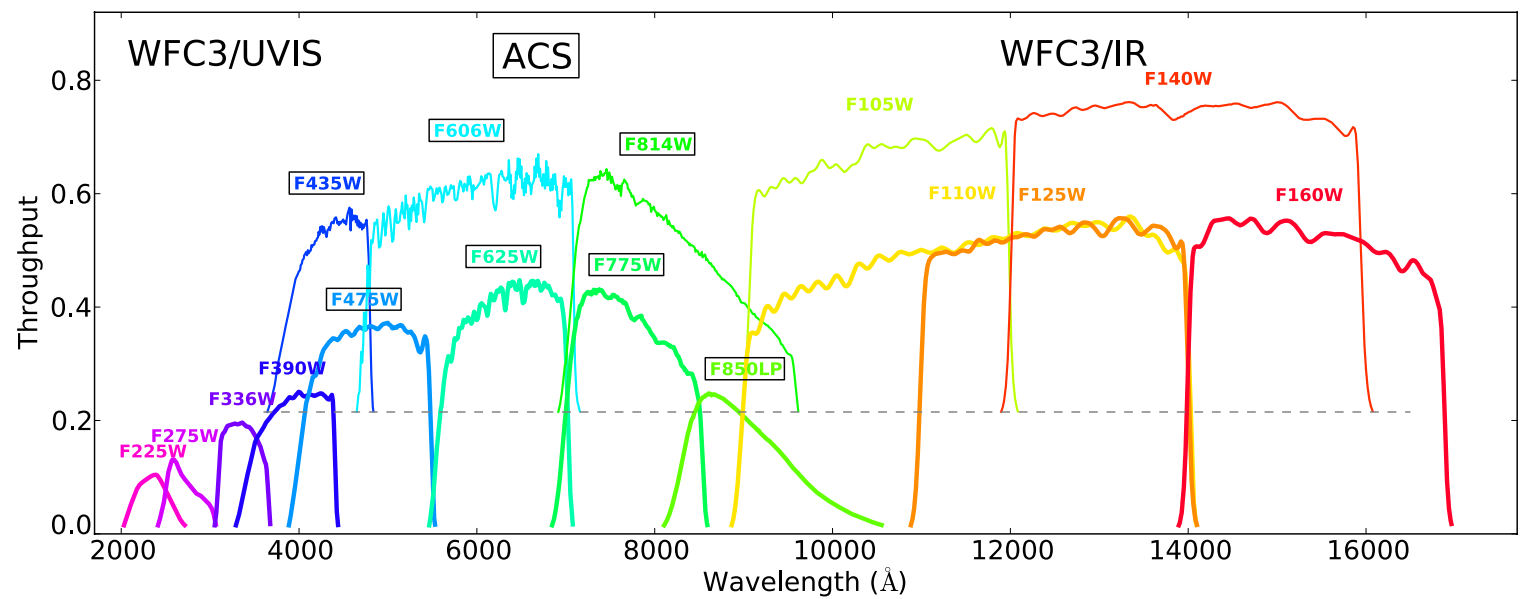

Figure 10. Each CLASH cluster is observed in 16 HST filters spanning 2000-17000 ̊ with WFC3/UVIS in the near-ultraviolet, ACS in the optical (extending into the near-infrared), and WFC3/IR in the near-infrared (see Table 5). Total throughput curves are plotted for each filter. For clarity, some curves are offset vertically by 0.2 (dashed line).

(A color version of this figure is available in the online journal.)

Table 5

CLASH Exposure Times, Limiting Magnitudes, and Extinction Coefficients

\begin{tabular}{lcccccc}
\hline \hline $\begin{array}{l}\text { Camera / } \\
\text { Channel }\end{array}$ & $\begin{array}{c}\text { Filter } \\
\text { Element }\end{array}$ & Orbits & $\begin{array}{c}\text { Average Exposure } \\
\text { Time }(\mathrm{s})^{\mathrm{a}}\end{array}$ & $\begin{array}{c}10 \sigma \text { Limit } \\
(\mathrm{AB} \mathrm{mag})^{\mathrm{b}}\end{array}$ & $\begin{array}{c}5 \sigma \text { Limit } \\
(\mathrm{AB} \text { mag })^{\mathrm{b}}\end{array}$ & $\begin{array}{c}\text { Galactic Ext. } \\
(\mathrm{AB} \text { mag/E} / E-V))\end{array}$ \\
\hline WFC3/UVIS & F225W & 1.5 & 3558 & 25.7 & 26.4 & 7.474 \\
WFC3/UVIS & F275W & 1.5 & 3653 & 25.7 & 26.5 & 6.140 \\
WFC3/UVIS & F336W & 1.0 & 2348 & 25.9 & 26.6 & 5.090 \\
WFC3/UVIS & F390W & 1.0 & 2350 & 26.5 & 27.2 & 4.514 \\
ACS/WFC & F435W & 1.0 & 1984 & 26.4 & 27.2 & 4.117 \\
ACS/WFC & F475W & 1.0 & 1994 & 26.8 & 27.6 & 3.724 \\
ACS/WFC & F606W & 1.0 & 1975 & 26.9 & 27.6 & 2.929 \\
ACS/WFC & F625W & 1.0 & 2008 & 26.4 & 27.2 & 2.671 \\
ACS/WFC & F775W & 1.0 & 2022 & 26.2 & 27.0 & 2.018 \\
ACS/WFC & F814W & 2.0 & 4103 & 27.0 & 27.7 & 1.822 \\
ACS/WFC & F850LP & 2.0 & 4045 & 2645 & 26.7 & 1.473 \\
WFC3/IR & F105W & 1.0 & 2415 & 27.0 & 27.3 & 1.015 \\
WFC3/IR & F110W & 1.0 & 2425 & 26.5 & 27.8 & 0.876 \\
WFC3/IR & F125W & 1.0 & 2342 & 26.7 & 27.4 & 0.757 \\
WFC3/IR & F140W & 1.0 & 4920 & 26.7 & 27.5 & 0.609 \\
WFC3/IR & F160W & 2.0 & & & & 0.470 \\
\hline
\end{tabular}

Notes.

${ }^{a}$ Exposure times are the average time for each filter for all CLASH cycle 18 observations. Exposure times for cycles 19 and 20 may differ slightly due to scheduling considerations.

${ }^{\mathrm{b}}$ Limiting magnitudes are for a circular aperture that is $0.4 \mathrm{arcsec}$ in diameter.

HST Program ID (where available), and the number of existing archival orbits available, respectively, for each cluster. The multiband observations span the near-ultraviolet to near-infrared (2000-17000 $\AA$ ). In the wavelength range $\sim 4000-9000 \AA$, we use ACS for its greater throughput efficiency (especially toward the red end) and larger observing area.

A typical CLASH observing sequence is presented in Table 6. Each cluster is observed at two orientations to increase the area covered in the parallel field SN search. The two orientation angles are typically $\sim 30^{\circ}$ apart to minimize the overlap between the ACS parallel pointings (see Section 4.3 for more details). Although we label the visits as "A" and "B" in Table 6, either orientation may be executed first. The choice depends solely on scheduling constraints. Indeed, there are often overlaps in time when the " $A$ " and " $B$ " orientations are both being executed. When the entire sequence of exposures for a cluster is completed, the region covered by all 16 filters subtends an area of $4.08 \mathrm{arcmin}^{2}$. Larger areas about the cluster center are covered by ACS in seven filters. The survey footprint and exposure map are shown in Figure 11. Color images of the co-added HST data from the first four CLASH clusters are shown in Figure 12.

CLASH clusters will be observed over the course of three annual HST observing cycles, with 10,10 , and 5 clusters to be observed in cycles 18,19 , and 20 , respectively. The current assignments of the cluster observations to the HST cycle number are shown in Column 5 of Table 3. The duration of this MCT program is driven, in part, by the cadence required by $\mathrm{SN}$ search (see Section 4.3 below).

\subsection{Filter Selection and Exposure Times}

Redshift estimates for multiply lensed images are crucial for breaking lensing degeneracies and tightening constraints on mass profiles (e.g., Broadhurst et al. 2005; Zitrin et al. 2009; Saha \& Read 2009). However, most of the useful lensed images are much too faint for spectroscopy. Typical 
Table 6

Typical CLASH Observing Sequence

\begin{tabular}{|c|c|c|c|c|c|}
\hline Visit ID & Epoch $^{\mathrm{a}}$ & $\begin{array}{l}\text { Primary } \\
\text { Camera }\end{array}$ & $\begin{array}{c}\text { Primary Cluster } \\
\text { Target Filters }\end{array}$ & $\begin{array}{l}\text { Parallel } \\
\text { Camera }\end{array}$ & $\begin{array}{l}\text { Parallel Field } \\
\text { Filters }\end{array}$ \\
\hline \multicolumn{6}{|c|}{ Orientation A } \\
\hline $\mathrm{A} 0, \mathrm{~A} 1$ & $1 \mathrm{~A}$ & ACS & $\begin{array}{l}\text { F625W, F850LP } \\
\text { F475W, F775W }\end{array}$ & WFC3 & F350LP, F125W, F160W \\
\hline $\mathrm{A} 2$ & $1 \mathrm{~A}$ & WFC3 & F110W, F160W & ACS & F775W, F850LP \\
\hline A3 & $2 \mathrm{~A}$ & ACS & F606W, F814W & WFC3 & F350LP, F125W, F160W \\
\hline A4 & $2 \mathrm{~A}$ & WFC3 & F110W, F160W & ACS & F775W, F850LP \\
\hline A5 & $3 \mathrm{~A}$ & ACS & F435W, F814W & WFC3 & F350LP, F125W, F160W \\
\hline $\mathrm{A} 6^{\mathrm{b}}$ & $3 \mathrm{~A}$ & WFC3 & F225W, F390W & ACS & F775W, F850LP \\
\hline A8 & $4 \mathrm{~A}$ & ACS & F814W, F850LP & WFC3 & F350LP, F125W, F160W \\
\hline A9 & $4 \mathrm{~A}$ & WFC3 & F110W, F160W & ACS & F775W, F850LP \\
\hline \multicolumn{6}{|c|}{ Orientation B } \\
\hline B0 & 1B & ACS & F625W, F850LP & WFC3 & F350LP, F125W, F160W \\
\hline B1 & 1B & WFC3 & F125W, F160W & ACS & F775W, F850LP \\
\hline B2 & $2 \mathrm{~B}$ & ACS & F606W, F775W & WFC3 & F350LP, F125W, F160W \\
\hline $\mathrm{B} 3^{\mathrm{b}}$ & $2 \mathrm{~B}$ & WFC3 & F275W, F390W & ACS & F775W, F850LP \\
\hline B5 & $3 \mathrm{~B}$ & ACS & F435W, F814W & WFC3 & F350LP, F125W, F160W \\
\hline B6 & 3B & WFC3 & F336W, F105W, F140W & ACS & F775W, F850LP \\
\hline B7 & 4B & ACS & F475W, F850LP & WFC3 & F350LP, F125W, F160W \\
\hline B8 & 4B & WFC3 & F125W, F160W & ACS & F775W, F850LP \\
\hline
\end{tabular}

Notes.

${ }^{\text {a }}$ Epochs $1 \mathrm{~A}-4 \mathrm{~A}$ are sequential in time, as are epochs $1 \mathrm{~B}-4 \mathrm{~B}$. However, depending on scheduling constraints, exposures for orientation $\mathrm{B}$ may precede or be interlaced with those for orientation $\mathrm{A}$.

b Visits A6 and B3 are each executed as a single two-orbit visit using the same guide star acquisition. This is essential to facilitate alignment of the NUV exposures.

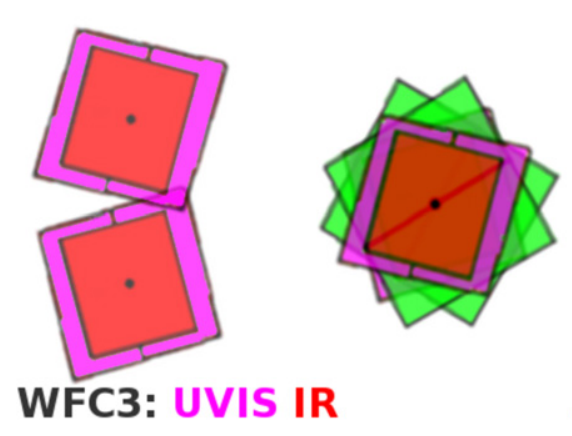

Figure 11. Each cluster is observed at two orientations approximately $30^{\circ}$ apart to increase the supernova search area in the parallel fields. WFC3 parallel observations are obtained while the cluster core is being observed with ACS and vice versa. At the median cluster redshift of $z_{\text {med }}=0.4$, the 6 arcmin separation between the center of the parallel field and the cluster center corresponds to a projected distance of $1.9 \mathrm{Mpc}$.

(A color version of this figure is available in the online journal.)

lensed source magnitudes are $23<I<28$ (Figure 13), so that only the brightest arcs yield spectroscopic redshifts even when observed with the largest ground-based facilities. With continuous sampling of the broad wavelength range from the NUV to NIR ( 2000-17000 $)$ ) that is enabled with WFC3 and ACS we can now obtain very accurate photometric redshifts (photo- $z$ 's) for most of the lensed objects down to an apparent F775W AB magnitude limit of 26.

For a fixed total observing time, splitting observations into multiple (ideally overlapping) filters significantly improves photo- $z$ precision (Benítez et al. 2009). We performed simulations to inform our filter selection and estimate our eventual photo- $z$ precision. Galaxy magnitudes, redshifts, and SEDs were drawn from the UDF (Coe et al. 2006) and "re-observed" with our filter set, adding noise as appropriate given our proposed depths in each filter. Photo- $z$ 's were then re-estimated using
BPZ (Benítez 2000; Benítez et al. 2004; Coe et al. 2006). In this simulation, we find that for 16 filters, we obtain very accurate $(\Delta z \sim 0.02(1+z))$ photo- $z$ 's for $80 \%$ of objects with F775W mag $<26$. Most importantly, we find that we will be able to acquire $\sim 6$ times as many reliable photometric redshifts than spectroscopic redshifts for objects at $z>1$, enabling a very substantial improvement in the number of unique constraints on the DM mass distributions (see Figure 13).

The coverage provided by the 16 WFC3 and ACS filters allows the Lyman-limit feature (rest frame $912 \AA$ ) to be photometrically traced to redshifts as low as $z \sim 1.5$ and Ly $\alpha$ to be detected out to $z \sim 10$. The inclusion of NUV photometry, for example, resolves one of the most common photo- $z$ degeneracies between the Balmer break in $z \sim 0.2$ galaxies and the Lyman break in $z \sim 3$ galaxies (Rafelski et al. 2009).

The exposure times for the primary camera (cluster center position) are set primarily by the need to achieve $80 \%$ photometric redshift completeness down to $\mathrm{F} 775 \mathrm{~W}=26 \mathrm{AB}$ mag. This requires $\sim 18$ orbits per cluster, as we need to achieve a $10 \sigma$ limiting AB magnitude of 26 in each filter. We augment this by two orbits per cluster to extend the NIR depth to a $10 \sigma$ limiting $\mathrm{AB}$ mag of $\mathrm{F} 160 \mathrm{~W}=26.7$ and $\mathrm{F} 814 \mathrm{~W}=27.0$ as needed for our lensed high- $z$ galaxy search (Section 2.3 and see Table 5). The limiting magnitudes in Table 5 are for a 0.4 diameter circular aperture and a point source with a flat $F_{v}$ spectrum. The minimum exposure in any given filter is one orbit and observations in each filter are distributed over at least four exposures to ensure robust cosmic ray rejection.

The five NIR filters provide the ability to identify $z>7$ galaxies with high confidence. Measured colors in these filters are essential for discriminating between such high-redshift galaxies and lower-redshift highly reddened galaxies (e.g., see Figure 14). The former are generally relatively flat in $F_{v}$ (or $\mathrm{AB}$ magnitudes) in the NIR while the latter generally increase in brightness as a function of wavelength. Quantitatively, the 

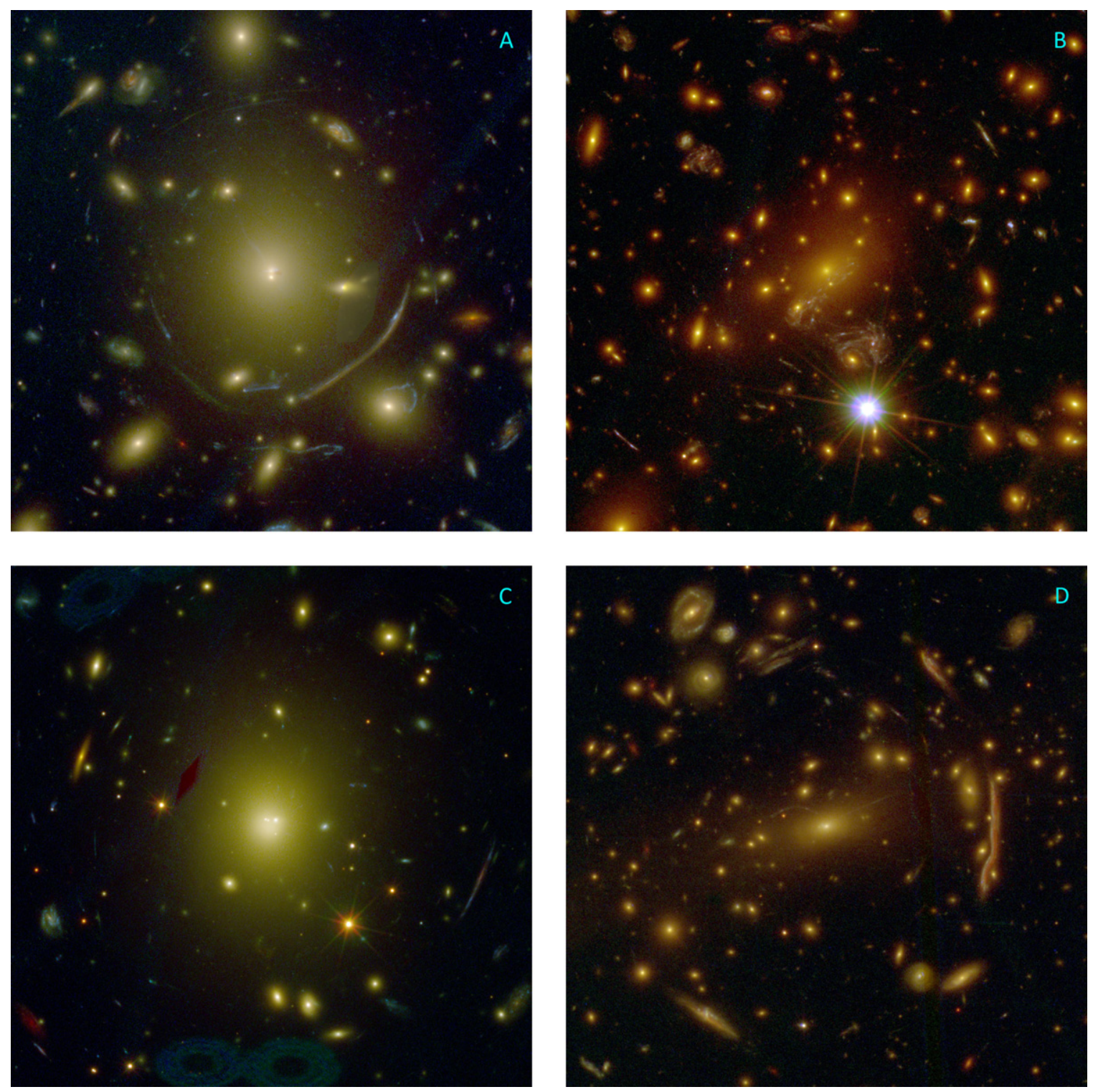

Figure 12. Cutouts of the CLASH images of the central regions of (A) A383, (B) MACS1149.6+2223, (C) A2261, and (D) MACS1206.2 - 0847 . The field of view shown in each image is $65 \times 65$ arcsec and is centered on the brightest cluster galaxy. These color composites are made from a combination of 12 of the 16 filters available for each cluster. The blue channel is a sum of the ACS F435W and F475W filters, the green channel is a sum of the ACS F606W, F625W, F775W, F814W, and F850LP filters, and the red channel is a sum of all five of the WFC3/IR broadband filters. Each cutout shown here represents $\sim 28 \%$ of the area covered by all 16 filters and just $\sim 10 \%$ of the total area imaged in the cluster core.

(A color version of this figure is available in the online journal.)

following selection criteria have proven effective: $z-J>0.8$ (a flux decrement factor of 2.1) and $J-H<0.5$ (Bradley et al. 2008, 2012; Zheng et al. 2009). To reliably measure the above colors for high- $z$ lensed galaxy detection, our exposures are set to reach the following $\mathrm{AB}$ magnitude limits: F850LP: $26.7(5 \sigma)$, F110W: $27.8(5 \sigma)$, F125W: $26.5(10 \sigma)$, and F160W $26.7(10 \sigma)$.

\subsection{Dither Pattern}

In each orbit we use a compact four-point dither pattern that provides half-pixel sampling along both detector axes. The dither pattern serves to both improve the spatial sampling of the point spread function (PSF), especially for the WFC3/IR detector with its large pixel scale of 0.128 arcsec pixel ${ }^{-1}$, and to help remove hot pixels and other detector imperfections that may be unaccounted for in the calibration reference files. In the first epoch of each roll angle, we use a small-scale dither pattern to preserve, as best as possible in light of significant geometric distortions, half-pixel sampling across the detector. In subsequent epochs involving WFC3/IR observations, either in prime or parallel, we use a slightly larger dither pattern to help identify and remove persistence artifacts from compact sources, which, if uncorrected, could possibly be misidentified as $\mathrm{SN}$ candidates. While our small-scale dither patterns are much smaller than is needed to cover the WFC3/UVIS and ACS/WFC detector gaps, our cluster observations are obtained at two orientations, leaving only two small diamond-shaped regions $\left(\sim 4.4 \operatorname{arcsec}^{2}\right.$ each $)$ in the central cluster area without data in all 16 filters.

\subsection{Observation Cadence and Supernova Follow-up}

Parallel observations are being obtained with a primary science goal of detecting SNe Ia. While the cluster core is being imaged with ACS, we obtain parallels with WFC3 and vice versa. For each of the two roll angles, observations are obtained over the course of four epochs spread out over $\sim 30-45$ days 


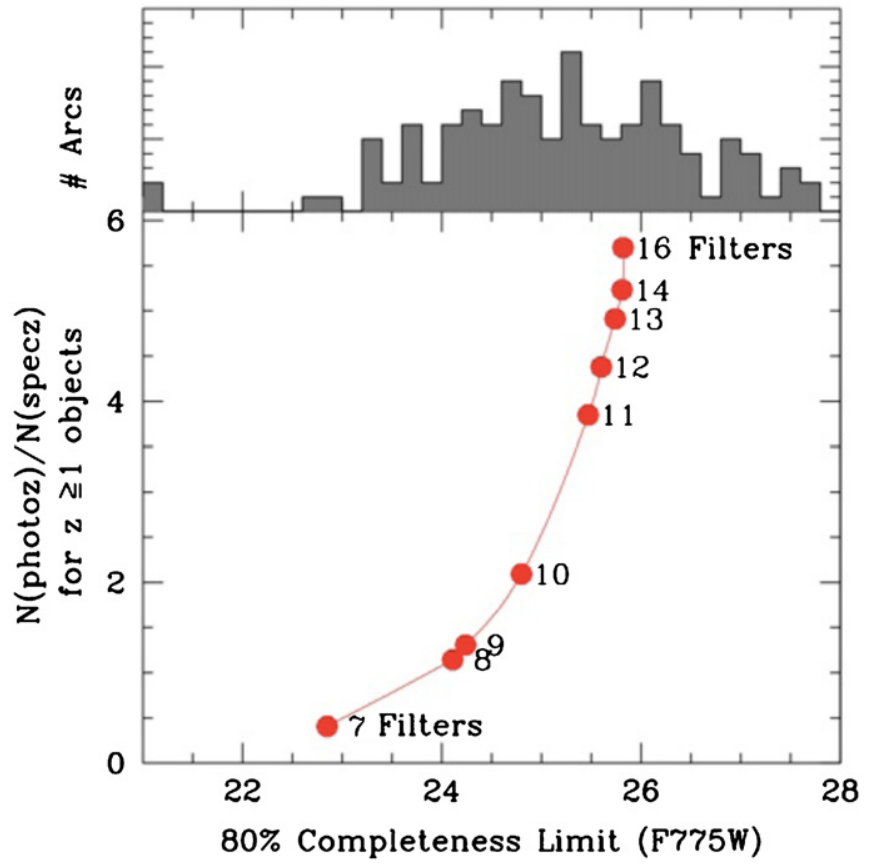

Figure 13. Top: magnitude distribution of 132 multiply lensed images detected in A1689 and CL0024+17 (Broadhurst et al. 2005; Zitrin et al. 2009). Most are too faint for spectroscopic follow-up. Bottom: by increasing the number of filters for a fixed observing time, we show how photometric redshifts are improved using two metrics. The horizontal axis shows, for example, that 16 filters yield very accurate $(\Delta z \sim 0.02(1+z))$ photo- $z$ 's for $80 \%$ of F775W mag $<26$ objects. This allows us to obtain precise redshifts for $\sim 6$ times more objects than are attainable using spectroscopy, as shown on the vertical axis. These estimates are based on simulated photometric and photo- $z$ catalogs derived from galaxies observed in the UDF (Coe et al. 2006).

(A color version of this figure is available in the online journal.)

( 10-14 days between observations). Image-differencing analysis enables us to detect $\mathrm{SNe}$ which have gone off between observations. As noted above, the exposure sequences are executed either sequentially or interleaved, depending on scheduling availability.

Upon detection of a promising high- $z$ SN Ia in the parallel field around a cluster, we can reprogram the remaining paral- lels to provide the follow-up (light-curve imaging and grism spectroscopy) necessary to measure its distance. The ability to reprogram the later of the two orientations to follow-up an SN detected early in a cluster observing sequence sets the upper limit on the angular offset between the two orientations-both orientations must be accessible during the entire cluster sequence. This constraint means that the two orientations cannot be more than $\sim 30^{\circ}$ apart. A lower limit on the angular separation between the two orientations is set to $\sim 20^{\circ}$ to ensure there is not excessive overlap in the ACS parallel pointings (with the exception of RXJ1347 where an orientation shift of $15^{\circ}$ was needed to avoid placing a bright star in the parallel fields). The ability to reprogram a downstream orientation shift means that a new target of opportunity (ToO) observation will not be required if the $\mathrm{SN}$ is discovered early in the cluster observing sequence. In this regard, the design of the CLASH SN program has part of the follow-up built into its implementation. For the flexibility required to follow $\mathrm{SNe}$ found at the end of their corresponding cluster observing sequence, a small number of ToOs (four) and follow-up reserve orbits are included in the CLASH orbit allocation. CLASH parallel fields are not observed in 16 filters. The ACS parallels are taken in F775W and F850LP and the WFC3 parallels are taken in F350LP (UVIS), F125W (IR) and F160W (IR) (see Table 6).

The CLASH and CANDELS (Grogin et al. 2011; Koekemoer et al. 2011) SN programs are tightly coordinated and, in fact, share a common pool of reserve orbits from which both programs can draw upon for follow-up. This common reserve has been allocated 200 orbits: 50 from the CLASH allocation and 150 from CANDELS. The coordinated program is led by A. Riess who is a co-I in both programs.

\subsection{ACS Failure Options}

While ACS functionality was restored in SM4, it is now only a "single-string" instrument, meaning there is no redundant path if the main CCD electronics box experiences a failure. This was a constraint imposed by the nature of the ACS repair. The primary impact of such a failure would be the loss of our parallel observations for $\mathrm{SN}$ searches. SNe could still be detected in our primary cluster core observations assuming we were to continue
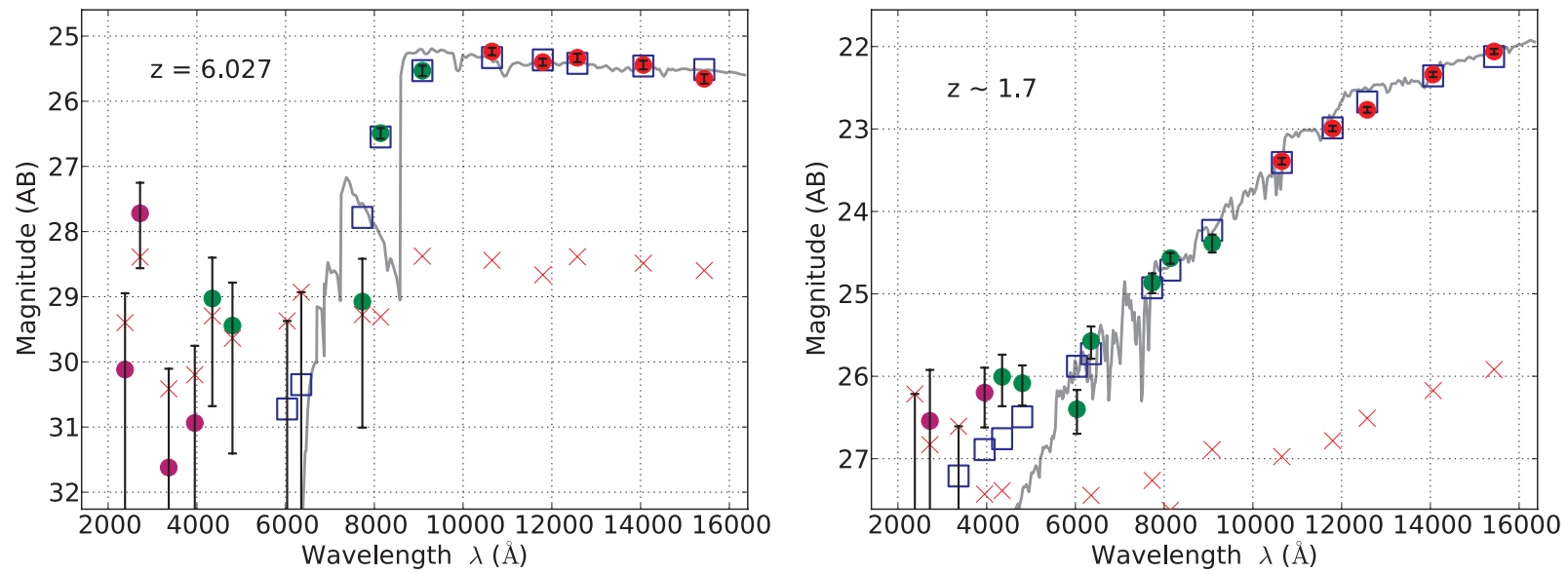

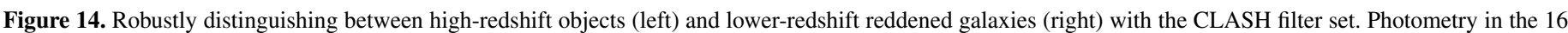

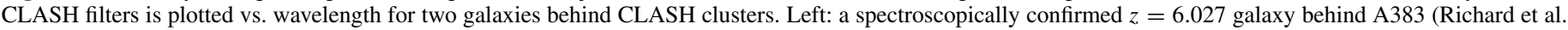

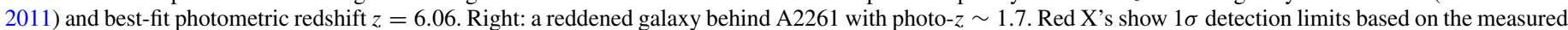

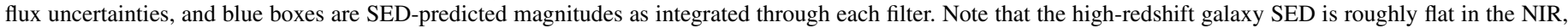

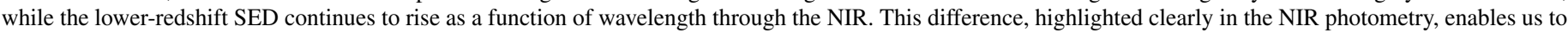
robustly distinguish high-redshift galaxies from lower-redshift interlopers.

(A color version of this figure is available in the online journal.) 
to distribute the exposures over multiple epochs. However, the use of SNe Ia for cosmology in strongly lensed regions is fraught with difficulty (see Section 2.2). While a failure of ACS would be a significant blow to the survey's dark energy science objectives, all other components of the CLASH science program, including our prime objective of studying the distribution of mass profile properties in clusters, would be able to be pursued with WFC3 alone.

If ACS fails permanently, we would abandon the dual orientation strategy and would, most likely, abandon the multiple epoch exposures, allowing the observations of each cluster to be completed on a much shorter time frame. By observing each cluster at a single roll angle, we would slightly increase the area in which we obtain full 16-filter coverage. All of the ACS filters are available with WFC3/UVIS, which would allow us to continue our 16-filter observations with WFC3 alone. However, there would be noticeable reductions in $\mathrm{S} / \mathrm{N}$ in the redder filters (F775W, F814W, F850LP). In order to continue to achieve the same depths, we would require exposure times in the redder filters to be increased by $80 \%$, adding about four orbits of integration time to each cluster.

The WFC3/UVIS and WFC3/IR fields of view are large enough to contain and yield robust photometric redshifts for most of the strongly lensed arcs. We would lose the extra areal coverage provided by the ACS observations, slightly reducing the area of overlap with our Subaru images. However, we estimate the impact on our ability to measure the mass profiles over the full radial range will be negligible.

\section{THE CLASH DATA PIPELINE}

The bulk of our HST data analyses make use of mosaics of globally aligned and co-added images. To accomplish this, we use the MosaicDrizzle pipeline (Koekemoer et al. 2002). The MosaicDrizzle pipeline takes as its input the calibrated FLT files which have been produced by calacs and calwf3. The ACS/WFC data, however, are first corrected for bias-striping and charge transfer efficiency (CTE) degradation effects (Anderson \& Bedin 2010). The CTE correction approximately reverses the effects of charge being trapped and trailed across the image during data readout. However, a similar procedure has yet to be developed and implemented for WFC3/UVIS images. We find that this uncorrected CTE can most significantly affect our UVIS photometry as follows. Trails from cosmic rays can leak into photometric apertures of non-detections, artificially boosting their observed fluxes. Objects otherwise expected to be UVIS dropouts, or non-detections (based on ACS+IR photometry), may commonly have significant detections in one or more UVIS filters. We find this can be greatly mitigated by adopting a more aggressive rejection of cosmic rays and their trails. The result is that most expected UVIS dropouts do actually drop out, though with some significant (as high as $\sim 5 \sigma$ ) detections remaining.

MosaicDrizzle then carries out a sequence of steps aimed at aligning the exposures in all the different camera/filter combinations within each visit and also across visits, using a combination of catalog matching and cross-correlation. The catalog matching is done using a deep ground-based catalog (typically from Subaru's SuprimeCam) as an initial reference point, followed by subsequent matching using catalogs from the CLASH HST images themselves, solving for shifts and rotations, which yields an accuracy of $0.1-0.2$ pixel. Shifts are further refined using cross-correlation, which can achieve accuracies to the level of $0.02-0.05$ pixel, thus $1-2$ mas for ACS images, limited essentially at that point by the stability of the PSF from one exposure to the next, as well as the accuracy of the distortion correction which is also removed from the images at this step.

The iterative shift refinement is then used to enable cosmic ray rejection to be carried out for all the exposures in a given filter across multiple epochs, leading to a final set of cosmic ray and bad pixel masks. These are then weighted according to the sky level in each input exposure (as modulated by the flat field/detected quantum efficiency variation across the detector for each filter), along with the readnoise and accumulated dark current, to form an inverse variance image for each exposure. The drizzle combination is then carried out using the inverse variance images as weights, using a square kernel as well as a "pixfrac" parameter typically set to 0.8 , which is chosen to be appropriate to the output image pixel scale and the number of dithers per filter. An advantage of this approach is that it involves only a single geometric operation, transforming the pixel values from the FLT files directly onto the output frame and avoiding any additional convolution.

It is worth noting that the inverse variance describes the expected noise in the absence of any correlated noise; in practice, the images do have some amount of correlated noise (due to the PSF being sampled by the detector pixels to begin with, as well as the single-step transformation onto the output image plane), but typically this is no more than $\sim 10 \%-15 \%$ of the noise that would be expected in the absence of any correlation, and cannot be reduced further without making the output image pixels larger. For each cluster, two sets of image mosaics are generated-one drizzled to a 30 mas pixel $^{-1}$ grid and one drizzled to a 65 mas pixel $^{-1}$ grid (for both ACS and WFC3). We register the images with north up, and the images are aligned so that the objects are sampled by the same pixels for all the filters.

We also run a redundant image processing pipeline that is built upon the framework developed for the ACS GTO pipeline APSIS (Blakeslee et al. 2003). For the CLASH application, APSIS was modified to handle the WFC3 detectors. The use of this second pipeline provides a useful verification of the photometry and astrometry as well as a redundant processing facility. The main difference between MosaicDrizzle and APSIS is that APSIS does not require an external astrometric reference catalog and can derive the relative image shifts from the HST data alone. To date, both pipelines appear to generate data of roughly similar quality with MosaicDrizzle producing slightly better alignment precision.

The SN detection pipeline has been developed jointly by the CLASH and CANDELS teams. It will be described in more detail in a separate paper. Briefly, the SN detection pipeline uses an image-differencing scheme to identify SN candidates. Extensive work has already been devoted to minimizing false positive detections. IR persistence is one potential source for false positives in both the $\mathrm{SN}$ and high- $z$ galaxy detection projects. To address this, a dark exposure is obtained in the Earth-occultation just preceding the first visit for all CLASH and CANDELS observations. This enables a mask to be generated that can flag any suspect pixels in the initial exposure of a CLASH visit. Subsequent orbits in the visit are flagged based on our actual data.

\subsection{Object Detection and Characterization}

SExtractor (Bertin \& Arnouts 1996) is used to detect objects and measure their photometry. We run SExtractor (version 2.5.0) in dual image mode, using a detection image created from a 


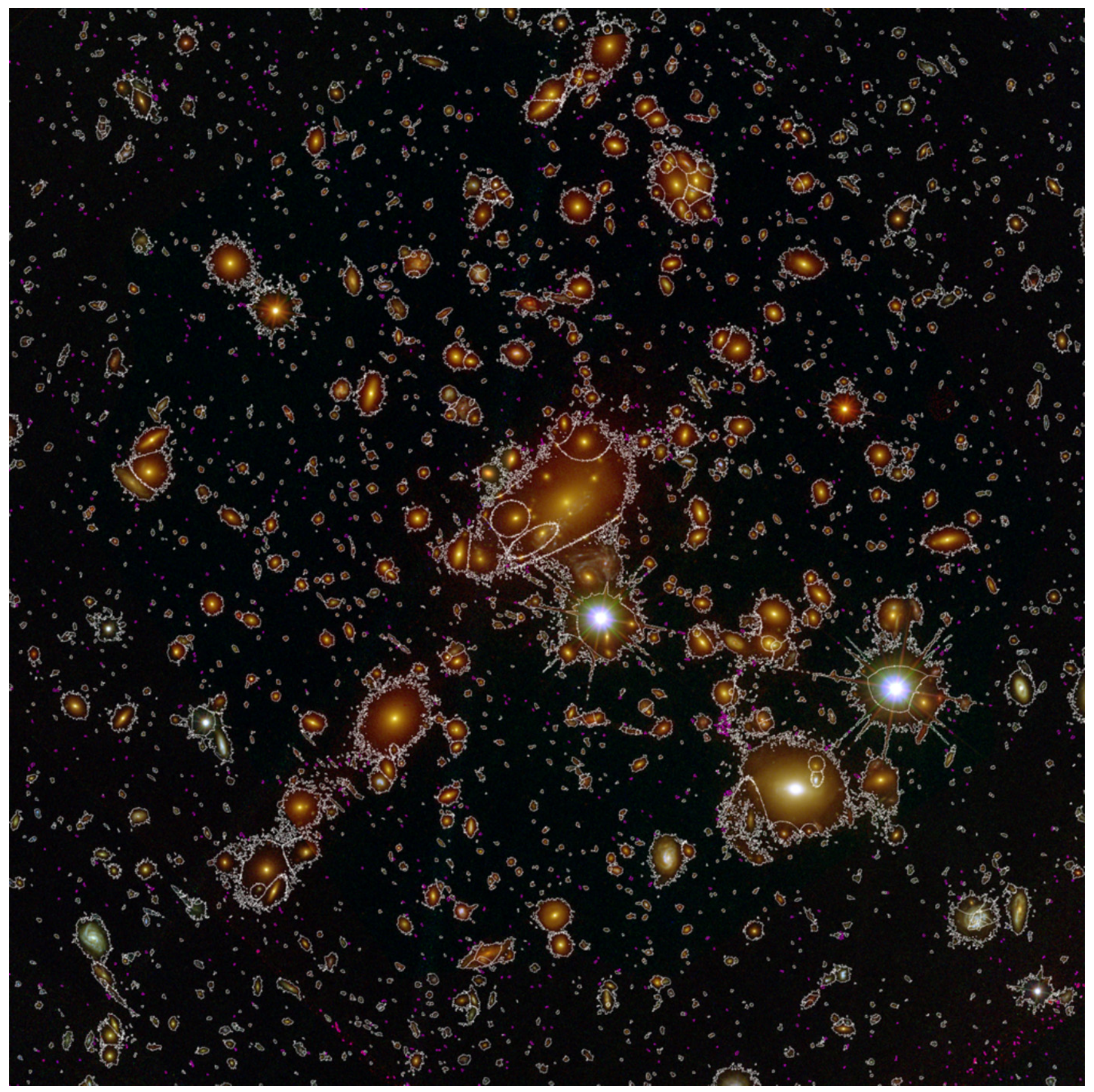

Figure 15. SExtractor segmentation map for the central region of the cluster MACS1149.6+2223. Detection of additional objects behind the cluster is accomplished by subtracting the best fits to the surface brightness distributions of the brightest of the cluster galaxies.

(A color version of this figure is available in the online journal.)

weighted sum of the ACS/WFC and WFC3/IR images. The weights come from the inverse variance images produced by Mosaicdrizzle. We do not use the WFC3/UVIS images in the construction of the detection image but, of course, run SExtractor on the UVIS data to compute source photometry. We also create a detection image solely from the WFC3/IR images to optimize the search for high-redshift $(z>6)$ objects.

For object detection in the ACS + IR images, we require a minimum of nine contiguous pixels at the level of the observed background rms or higher. The detection phase background sky level is computed in $5 \times 5$ grids of cells, with each cell being $128 \times 128$ pixels. For photometry, the local sky is estimated from a 24 pixel wide rectangular annulus around each detected object. The deblender minimum contrast ratio and number of threshold levels are 0.0015 and 32, respectively. These parameters were chosen after a systematic investigation of possible values and yields reasonable performance in minimizing spurious detections and suppressing overdeblending of bright objects, while achieving reasonable completeness in detecting faint sources behind bright cluster galaxies. The segmentation map for the cluster MACS1149.6+2223 using the above parameters is shown in Figure 15.

Our image processing effectively rejects cosmic rays from the central regions of our UVIS and ACS images where we have four or more overlapping exposures. However, such rejection is not possible in the corners and edges of our images, where we 
have fewer exposures due to our observing strategy (two roll angles and dithering). As each cosmic ray only affects a single observation, it will often only be detected in a single filter. We prune these detections from our catalog by rejecting any object with only a single $5 \sigma$ detection in one UVIS/ACS filter, as measured by SExtractor. We also reject any object without any $5 \sigma$ detections.

We perform a separate IR-based detection with more aggressive deblending (64 levels of 0.0001 minimum contrast) and background subtraction $(3 \times 3$ grids of $64 \times 64$ pixels $)$. This detection is slightly more sensitive to redder objects, including those at high-redshift. It also performs slightly better at deblending these fainter objects, including those at high- $z$ as well as arcs (strongly lensed galaxies), from brighter nearby cluster galaxies. As all WFC3/IR observations are obtained with multiple readouts and cosmic ray rejection, and to preserve our sensitivity to faint high-redshift objects, we do not prune this catalog based on $5 \sigma$ detections.

Automated detection of strongly lensed galaxies is often challenging due to crowding in the cluster core, especially for low-surface brightness galaxies stretched into long, thin arcs. Progress has been made (e.g., Seidel \& Bartelmann 2007), and we are testing this method for use with CLASH data. Currently, manual intervention is still required for those arcs which elude detection. We compare the initial source list with arc candidates identified both visually and based on our strong lens modeling. For arcs that are either missing from the detection list or that are only partially detected, we construct manual photometric apertures. We force SExtractor to adopt these apertures using the software package SExSeg (Coe et al. 2006). Arc photometry is then derived from images in which light from the BCG has been modeled and subtracted.

Isophotal apertures are used as they have been shown to yield robust colors (Benítez et al. 2004). In the source list presented in Section 5.3, no aperture corrections have been applied to the magnitudes. The PSF FWHM's span $\sim 0{ }^{\prime} 07-00^{\prime} 15$ (Ford et al. 2003; Sirianni et al. 2003; Dressel 2010). The photometric corrections for PSF variation are small for extended objects for all but the faintest sources, in part because we use relatively large isophotal apertures.

Extinction corrections are derived from the Schlegel et al. (1998) IR dust emission maps, and the resulting $A_{\lambda}$ coefficients, in $\mathrm{AB}$ mag per unit $E(B-V)$, are given in Table 5. Flux uncertainties are derived by SExtractor using the inverse variance images. We have compared the flux errors so derived with the predicted uncertainties from the ACS and WFC3 exposure time calculator and find excellent agreement.

\subsection{Photometric Redshifts}

We derive photometric redshift estimates using two independent packages: BPZ (Benítez 2000; Benítez et al. 2004; Coe et al. 2006) and LePHARE (Ilbert et al. 2006, hereafter LPZ). Both software packages use $\chi^{2}$ minimization and template fitting but differ in their specific templates and their assumed priors. LPZ uses the SED library optimized for the COSMOS survey (Ilbert et al. 2009) without template interpolation. BPZ currently uses PEGASE SED templates (Fioc \& Rocca-Volmerange 1997) which have been heavily recalibrated based on the FIREWORKS spectroscopic and photometric catalog (Wuyts et al. 2008). BPZ allows for interpolation between adjacent templates and uses an empirically derived prior on redshift and type based on observed magnitude. A full analysis of the accuracy and precision of our photometric redshift estimates will be discussed in an upcoming paper (S. Jouvel et al. 2012, in preparation). The photo- $z$ accuracy analyses will be based on over 1000 redshifts, obtained from the facilities described in Section 6.

\subsection{Source List for MACS1149.6+2223}

We present here an initial version of a representative CLASH source list for the $z=0.544$ cluster MACS1149.6 +2223 . The full list is available online (at MAST treasury program archive Web site) and has over 100 parameters per object. In Table 7, we present just a small subset of the full list: 100 galaxies within 1 arcmin of the cluster center. This source list is based on the $\mathrm{ACS}+\mathrm{WFC} 3 / \mathrm{IR}$ detection image (see Section 5.1). Column 1 gives the SExtractor object ID, Columns 2 and 3 give the equatorial coordinates (J2000), Column 4 gives the isophotal area of the detection (in 0 '.065 pixels), Column 5 gives the ellipticity of the source as measured by SExtractor, Column 6 gives the number of passbands in which the object is detected at a significance of $5 \sigma$ or greater, and Column 7 gives the total number of bands in which the object is detected. In the case of MACS1149.6 +2223, there are 17 HST passbands available because the archival imaging included ACS F555W. Note that even bright sources will sometimes not be detected in all bands because the footprints of each detector on the sky are different (see Figure 11). Columns 8-10 give the BPZ photometric redshift estimate, the BPZ Odds parameter (Benítez et al. 2004), and the $\chi^{2}$ measurement presented in Coe et al. (2006). The $95 \%$ confidence limits on the range of the photometric redshift estimate are given in the row just below the best-fit photometric redshift value. This range is an indicator of the uncertainty in the redshift value. In general, BPZ photo- $z$ estimates with Odds $>0.90$ are the most reliable estimates. Figure 16 shows the histogram of the BPZ photometric redshifts for 232 galaxies in the cluster MACS1149.6 +2223 with BPZ Odds $\geqslant 0.90$, F850LP magnitude $\leqslant 26.5$ and $z_{\text {phot }} \leqslant 3$. The histogram shows the cluster peak very clearly.

The remaining columns in Table 7 give the isophotal magnitudes (corrected for Galactic extinction) for five of the CLASH passbands: F275W, F390W, F850LP, F125W, and F160W. The photometric error in the isophotal magnitude is given, in parentheses, in the row directly below the magnitude value. Photometry has been corrected for Galactic extinction using an $E(B-V)=0.02297$, and all magnitudes given are on the AB photometric system (see Table 5 for extinction coefficients used). A magnitude value of 99 indicates a non-detection (negative flux) in that band. In this case, the magnitude error value gives the $1 \sigma$ detection limit in that band. A magnitude value of -99 indicates that the source lies outside of the detector field of view or lies within a gap between detectors. The full source lists for each CLASH cluster are released, via MAST, $\sim 6$ months after the final observation for that cluster is acquired.

\section{SUPPORTING OBSERVATIONS}

As discussed above, having both weak and strong-lensing information as well as information about the cluster baryonic mass distribution are critical for deriving robust mass profiles and concentrations. A partial list of the supporting multiwavelength imaging being used in the CLASH program is summarized in Table 8. All CLASH clusters have X-ray imaging as well as wide-field multiband ground-based optical imaging. The X-ray imaging is from Chandra/ACIS (Garmire et al. 2003) and some of the clusters have XMM/EPIC (Strüder et al. 2001; Turner et al. 2001) imaging as well. 
Table 7

Sample of CLASH Source List for MACS1149.6+2223

\begin{tabular}{|c|c|c|c|c|c|c|c|c|c|c|c|c|c|c|}
\hline ID & $\alpha_{\mathrm{J} 2000}$ & $\delta_{\mathrm{J} 2000}$ & $\begin{array}{c}\text { Area } \\
\text { (pixels) }\end{array}$ & Ell & Nsig5 & Nfobs & $\begin{array}{c}\mathrm{zbpz} \\
{[\min , \max ]}\end{array}$ & Odds & $\chi^{2}$ & $\begin{array}{c}\text { F275W } \\
\text { (err) }\end{array}$ & $\begin{array}{c}\text { F390W } \\
\text { (err) }\end{array}$ & $\begin{array}{l}\text { F850LP } \\
\text { (err) }\end{array}$ & $\begin{array}{c}\text { F125W } \\
\text { (err) }\end{array}$ & $\begin{array}{c}\text { F160W } \\
\text { (err) }\end{array}$ \\
\hline 4756 & 177.4090 & 22.4002 & 9 & 0.36 & 4 & 17 & $\begin{array}{l}2.990 \\
{[2.73,3.09]}\end{array}$ & 0.63 & 1.32 & $\begin{array}{l}29.64 \\
(1.50)\end{array}$ & $\begin{array}{l}29.05 \\
(0.65)\end{array}$ & $\begin{array}{l}27.18 \\
(0.22)\end{array}$ & $\begin{array}{l}28.65 \\
(0.52)\end{array}$ & $\begin{array}{l}28.10 \\
(0.30)\end{array}$ \\
\hline 4761 & 177.3900 & 22.3999 & 65 & 0.47 & 12 & 17 & $\begin{array}{l}1.701 \\
{[1.62,1.75]}\end{array}$ & 0.94 & 2.44 & $\begin{array}{l}26.85 \\
(0.49)\end{array}$ & $\begin{array}{l}26.73 \\
(0.24)\end{array}$ & $\begin{array}{l}26.87 \\
(0.39)\end{array}$ & $\begin{array}{l}25.42 \\
(0.08)\end{array}$ & $\begin{array}{l}25.65 \\
(0.08)\end{array}$ \\
\hline 4762 & 177.3900 & 22.4002 & 10 & 0.45 & 4 & 17 & $\begin{array}{l}1.717 \\
{[1.49,1.77]}\end{array}$ & 0.59 & 0.63 & $\begin{array}{l}29.20 \\
(1.53)\end{array}$ & $\begin{array}{l}28.16 \\
(0.48)\end{array}$ & $\begin{array}{l}27.16 \\
(0.33)\end{array}$ & $\begin{array}{l}26.82 \\
(0.17)\end{array}$ & $\begin{array}{l}27.11 \\
(0.19)\end{array}$ \\
\hline 4763 & 177.3900 & 22.4000 & 8 & 0.42 & 1 & 17 & $\begin{array}{c}0.765 \\
{[0.76,2.22]}\end{array}$ & 0.12 & 0.63 & $\begin{array}{l}99.00 \\
(28.12)\end{array}$ & $\begin{array}{l}28.19 \\
(0.44)\end{array}$ & $\begin{array}{l}27.28 \\
(0.32)\end{array}$ & $\begin{array}{l}27.26 \\
(0.21)\end{array}$ & $\begin{array}{l}27.36 \\
(0.20)\end{array}$ \\
\hline 4764 & 177.3910 & 22.4002 & 29 & 0.27 & 12 & 17 & $\begin{array}{l}1.222 \\
{[1.12,1.32]}\end{array}$ & 0.63 & 1.98 & $\begin{array}{l}99.00 \\
(28.01)\end{array}$ & $\begin{array}{l}27.69 \\
(0.34)\end{array}$ & $\begin{array}{l}26.44 \\
(0.18)\end{array}$ & $\begin{array}{l}26.24 \\
(0.10)\end{array}$ & $\begin{array}{c}26.26 \\
(0.09)\end{array}$ \\
\hline 4765 & 177.4080 & 22.4002 & 15 & 0.16 & 8 & 17 & $\begin{array}{l}0.745 \\
{[0.67,4.03]}\end{array}$ & 0.31 & 1.47 & $\begin{array}{l}99.00 \\
(28.04)\end{array}$ & $\begin{array}{l}99.00 \\
(28.89)\end{array}$ & $\begin{array}{l}26.59 \\
(0.19)\end{array}$ & $\begin{array}{l}26.94 \\
(0.18)\end{array}$ & $\begin{array}{l}26.85 \\
(0.14)\end{array}$ \\
\hline 4773 & 177.4140 & 22.4002 & 4 & 0.31 & 1 & 17 & $\begin{array}{l}1.150 \\
{[1.25,2.41]}\end{array}$ & 0.14 & 0.87 & $\begin{array}{c}28.89 \\
(0.95)\end{array}$ & $\begin{array}{c}27.87 \\
(0.25)\end{array}$ & $\begin{array}{l}27.97 \\
(0.39)\end{array}$ & $\begin{array}{l}28.95 \\
(0.60)\end{array}$ & $\begin{array}{l}28.03 \\
(0.27)\end{array}$ \\
\hline 4778 & 177.3880 & 22.3999 & 152 & 0.13 & 12 & 17 & $\begin{array}{c}0.453 \\
{[0.43,0.47]}\end{array}$ & 0.98 & 4.00 & $\begin{array}{l}25.34 \\
(0.20)\end{array}$ & $\begin{array}{l}26.62 \\
(0.30)\end{array}$ & $\begin{array}{l}23.78 \\
(0.04)\end{array}$ & $\begin{array}{l}23.48 \\
(0.02)\end{array}$ & $\begin{array}{l}23.30 \\
(0.01)\end{array}$ \\
\hline 4780 & 177.4130 & 22.4000 & 260 & 0.03 & 13 & 17 & $\begin{array}{l}0.685 \\
{[0.66,0.72]}\end{array}$ & 0.91 & 5.22 & $\begin{array}{l}26.26 \\
(0.53)\end{array}$ & $\begin{array}{l}26.32 \\
(0.29)\end{array}$ & $\begin{array}{l}23.35 \\
(0.03)\end{array}$ & $\begin{array}{l}23.23 \\
(0.02)\end{array}$ & $\begin{array}{l}23.00 \\
(0.01)\end{array}$ \\
\hline 4782 & 177.3890 & 22.4001 & 8 & 0.06 & 2 & 17 & $\begin{array}{c}2.344 \\
{[2.20,2.76]}\end{array}$ & 0.32 & 0.28 & $\begin{array}{l}99.00 \\
(28.58)\end{array}$ & $\begin{array}{l}28.35 \\
(0.36)\end{array}$ & $\begin{array}{l}28.67 \\
(0.67)\end{array}$ & $\begin{array}{l}27.85 \\
(0.25)\end{array}$ & $\begin{array}{c}27.07 \\
(0.11)\end{array}$ \\
\hline
\end{tabular}

Notes. The complete source list with over 100 parameters per source is available online from IOP and from the MAST CLASH Treasury Archive Web site at http://archive.stsci.edu/prepds/clash/. All magnitudes are on AB system.

(This table is available in its entirety in a machine-readable form in the online journal. A portion is shown here for guidance regarding its form and content.)

Table 8

Partial List of Supporting Observations for CLASH at Time of Publication

\begin{tabular}{|c|c|c|c|c|c|c|}
\hline Cluster & $\begin{array}{c}\text { Proj. FOV of } \\
\text { Suprime Cam } \\
\quad(\mathrm{Mpc})\end{array}$ & $\begin{array}{l}\text { Suprime Cam } \\
\text { Filters }\end{array}$ & $\begin{array}{c}\text { Chandra } \\
\text { (ksec) }\end{array}$ & $\begin{array}{l}\text { XMM } \\
\text { (ksec) }\end{array}$ & $\begin{array}{c}\text { Spitzer }^{\mathrm{a}} \\
(\mathrm{ksec})\end{array}$ & $\begin{array}{c}\text { Bolocam } \\
(\mathrm{ksec})\end{array}$ \\
\hline \multicolumn{7}{|l|}{ X-ray Selected Clusters: } \\
\hline Abell 209 & 5.68 & BVRIZ & 20 & $\cdots$ & $(31.2)^{\mathrm{b}}$ & 66 \\
\hline Abell 383 & 5.26 & BVRIZ & 50 & $\ldots$ & 20.4 & 91 \\
\hline MACS0329.7-0211 & 9.68 & BVRZ & 70 & $\cdots$ & $(31.2)^{\mathrm{b}}$ & 39 \\
\hline MACS0429.6-0253 & 9.30 & VRI & 24 & $\ldots$ & $(31.2)^{\mathrm{b}}$ & 62 \\
\hline MACS0744.9+3927 & 11.90 & BVRIZ & 90 & 138 & 18.0 & 62 \\
\hline Abell 611 & 7.28 & BVRIZ & 40 & 28 & 18.0 & 79 \\
\hline MACS1115.9+0129 & 8.34 & BVRIZ & 56 & $\cdots$ & $(31.2)^{\mathrm{b}}$ & 56 \\
\hline Abell 1423 & 5.82 & VI & 36 & 31 & $\cdots$ & $\mathrm{TBA}^{\mathrm{d}}$ \\
\hline MACS1206.2-0847 & 9.56 & BVRIZ & 24 & $\cdots$ & $(31.2)^{\mathrm{b}}$ & 41 \\
\hline CLJ1226.9+3332 & 13.04 & BVZ & 75 & 120 & 17.0 & 68 \\
\hline MACS1311.0-0310 & 10.20 & $\mathrm{R}$ & 85 & $\cdots$ & $(31.2)^{\mathrm{b}}$ & 41 \\
\hline RXJ1347.5-1145 & 9.68 & BVRIZ & 185 & 36 & 19.2 & 59 \\
\hline MACS1423.8+2404 & 10.72 & BVRIZ & 140 & $\cdots$ & 21.4 & 85 \\
\hline RXJ1532.9+3021 & 8.22 & BVRIZ & 40 & $\cdots$ & 20.2 & 53 \\
\hline MACS1720.3+3536 & 8.90 & BVRIZ & 60 & $\cdots$ & 20.0 & 68 \\
\hline Abell 2261 & 6.06 & BVR & 35 & 33 & 22.4 & 46 \\
\hline MACS1931.8-2635 & 8.34 & BVRIZ & 114 & $\ldots$ & $(31.2)^{\mathrm{b}}$ & 63 \\
\hline RXJ2129.7+0005 & 6.26 & BVRIZ & 40 & $\cdots$ & 18.2 & 49 \\
\hline MS2137-2353 & 7.70 & BVRIZ & 150 & $\cdots$ & 20.4 & 47 \\
\hline RXJ2248.7-4431 (Abell 1063S) & $\cdots$ & $\begin{array}{c}\text { Not accessible } \\
\text { See note } c\end{array}$ & 26 & $\cdots$ & 20.4 & 20 \\
\hline \multicolumn{7}{|l|}{ High Magnification Clusters: } \\
\hline MACS0416.1-2403 & 9.30 & BRZ & 16 & $\cdots$ & $(31.2)^{\mathrm{b}}$ & 29 \\
\hline MACS0647.8+7015 & 11.10 & BVRIZ & 40 & $\cdots$ & 18.0 & 43 \\
\hline MACS0717.5+3745 & 10.76 & BVRIZ & 104 & $\cdots$ & 21.4 & 47 \\
\hline MACS1149.6+2223 & 10.72 & BVRIZ & 20 & $\cdots$ & 18.0 & 45 \\
\hline MACS2129.4-0741 & 10.96 & BVRIZ & 40 & $\ldots$ & 19.5 & 47 \\
\hline
\end{tabular}

Notes. Observations with MUSTANG (NRAO/GBT) pending. Spectroscopic observations summarized in text.

${ }^{a}$ Times shown are the total for both IRAC channels 1,2 (3.6 and 4.5 microns).

$\mathrm{b}$ These 8 clusters are being observed with the indicated exposures in Spitzer cycle 8.

${ }^{c}$ RXJ2248.7-4431 has UBVRIZ imaging with the WFI on the 2.2-meter telescope at La Silla. Total exposure times in each band are 10-11 hours.

${ }^{\mathrm{d}}$ SZE data for A1423 will be acquired in spring 2012. 


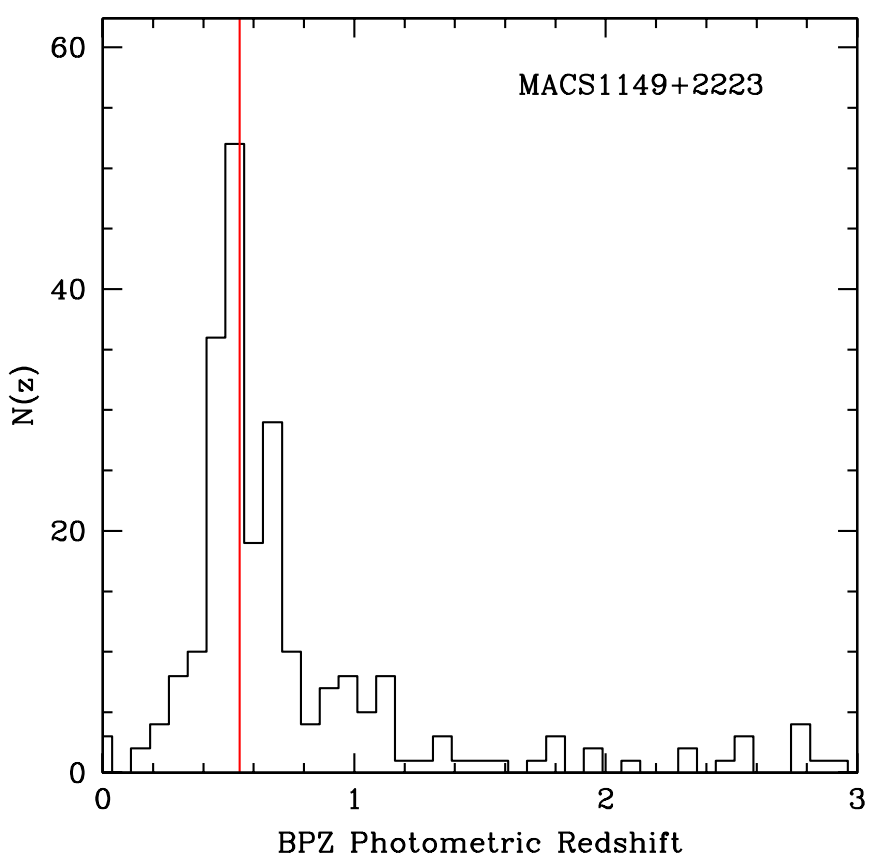

Figure 16. Histogram of the BPZ photometric redshifts for galaxies within 1 arcmin of the center of the cluster MACS1149.6+2223. The 232 galaxies included here have BPZ Odds $\geqslant 0.90$, F850LP magnitude $\leqslant 26.5$ and $z_{\text {phot }} \leqslant 3$. The histogram shows the cluster peak very clearly. The mean spectroscopic redshift of the cluster $(z=0.544)$ is denoted by the vertical red line. The average photometric redshift in the range $0.4 \leqslant z_{\text {phot }} \leqslant 0.65$ is $\left\langle z_{\mathrm{ph}}\right\rangle=0.515$. The accuracy of our photometric redshifts is expected to improve further as our spectroscopic sample increases. See S. Jouvel et al. (2012, in preparation) for details.

(A color version of this figure is available in the online journal.)

The ground-based optical imaging is, for 24 out of the 25 clusters, from the Subaru Observatory (and, in particular, performed with the SuprimeCam instrument; Miyazaki et al. 1998). The Subaru SuprimeCam imaging is currently available in three or more filters for 22 of the 24 clusters reachable from Mauna Kea, and we are pursuing additional optical filter coverage for the two remaining clusters. Wide-field optical imaging for A2261 from Subaru, for instance, was supplemented with Sloan Digital Sky Survey (SDSS) iz band imaging from the Kitt Peak $4 \mathrm{~m}$ Mayall telescope using the Mosaic1 camera. RXJ2248.7-4431 is too far south to reach with Subaru but has multiband imaging from the wide-field imager (WFI) on the European Southern Observatory's $2.2 \mathrm{~m}$ telescope. The multiband optical imaging enables robust selection of background galaxies for weak-lensing analysis (e.g., Medezinski et al. 2007).

We have also acquired millimeter-wave imaging to map the Sunyaev-Zel'dovich Effect (SZE) in order to measure the integrated line-of-sight gas pressure toward each cluster. The SZE data come primarily from two facilities-the Bolocam instrument at the Caltech Submillimeter Observatory (Golwala et al. 2009; Sayers et al. 2011) and Multiplexed SQUID/TES Array at Ninety Gigahertz (MUSTANG; Dicker et al. 2008, 2009) at the Robert C. Byrd Green Bank Telescope. All 25 CLASH clusters have a detected SZE signal with the lowest $\mathrm{S} / \mathrm{N} \sim 7$ and most with $\mathrm{S} / \mathrm{N}>10$. The combination of the $\mathrm{X}$-ray and millimeter-wave observations allows the mass scaling relations to be accurately calibrated for use in cosmological surveys (e.g., Okabe et al. 2010b). The X-ray and SZE data will be used to measure gas density profiles for subtraction from lensing-derived total mass profiles to yield DM-only mass profiles (e.g., Lemze et al. 2008). These data also allow assump- tions of hydrostatic equilibrium to be tested and constraints on thermal motion to be made from measurements of excess pressure (Umetsu et al. 2009; Zhang et al. 2010; Molnar et al. 2010).

Spitzer Space Telescope (Werner et al. 2004) data are available for most of the CLASH clusters from the IRAC Lensing Survey (PI: E. Egami). The few remaining clusters that lacked sufficiently deep Spitzer data will be observed in our cycle 8 ICLASH program (PI: R. Bouwens). The Spitzer data are critical for characterizing the high- $z$ galaxy population (e.g., see Richard et al. 2011). Deep IRAC (Fazio et al. 2004) imaging samples the rest-frame optical in high- $z$ galaxies, allowing us to perform stellar population modeling and derive stellar masses, and enables robust discrimination between star-forming $z>7$ galaxies and dusty/evolved galaxies at $z \sim 2$.

We have also been awarded $225 \mathrm{hr}$ of time on the Very Large Telescope as part of a large program (PI: P. Rosati) to obtain VIMOS spectroscopy of the 14 southern clusters $(\delta<+1.5)$. In addition, we are acquiring cluster and lensed galaxy redshifts from spectrographs on Magellan (GISMO; LDSS), LBT (MODS), MMT (Hectospec), and Palomar (double spectrograph).

\section{SUMMARY}

The CLASH will produce a major advance in our understanding of the DM power spectrum and the internal structure of cluster halos on scales from $10 \mathrm{kpc}$ to $2 \mathrm{Mpc}$. The precision to which these measurements are being made will provide an unprecedented foil against which we will challenge and ultimately expand our current ideas about structure formation and the nature of dark energy. In 2010 November, the CLASH MCT program initiated its three year observing plan to obtain deep (20-orbit) 16-band HST imaging for each of 25 galaxy cluster cores. The clusters in our sample are massive $\left(5 \times 10^{14}<M_{\mathrm{vir}} / M_{\odot}<3 \times 10^{15}\right)$, span a range of (intermediate) redshifts $(0.18<z<0.9)$, and most (20 of the 25) were selected based on their X-ray properties (reasonably relaxed and $T_{x}>5 \mathrm{keV}$ ). The 16-band $H S T$ imaging yields precise $(2 \%(1+z))$ photometric redshifts for all galaxies brighter than F775W AB mag 26, including hundreds of strongly lensed galaxies. When combined with weak-lensing maps from widefield Subaru imaging and constraints on the baryonic gas distribution from millimeter-wave and X-ray imaging, CLASH data will allow the cluster mass profiles to be tightly constrained over a large range of scales, yielding robust measurements of their central density concentrations. We also expect to provide important constraints on the degree of substructure in the DM distribution by studying galaxy-scale lensing. The strong lens magnifying power of our clusters should also enable detection of dozens of relatively bright $(m<26 \mathrm{AB}) z>7$ galaxies, including some bright enough for spectroscopic follow-up with ground-based telescopes and, certainly, many that can be studied with the spectrographs on James Webb Space Telescope. Parallel observations may detect up to $\sim 30 z>1$ SNe Ia that, when combined with $z>1$ SNe Ia detected by the CANDELS program, will provide new constraints on the time dependence of $w$ and a dramatic improvement in our understanding of SNe Ia evolution in the matter-dominated universe $(z>1.5)$.

CLASH data will also provide the mass calibrators for the next generation of big cosmological surveys such as the Dark Energy Survey (DES), Sunyaev-Zel'dovich surveys (e.g., the South Pole Telescope), and next generation X-ray cluster surveys. The DES will measure the evolution of the space density of 
clusters (among other probes) as a way to measure dark energy. These cluster counts tell us much about cosmological parameters through their impact on both the volume and the growth of perturbations. To do so, however, requires that the cluster mass scaling relations be well calibrated. The CLASH measurements of the enclosed mass in each cluster will be invaluable in calibrating the relation between the cluster mass and a number of observable mass proxies.

MCT programs represent a very large investment of HST observing time. These data are intended to be a community resource, and as such they have no proprietary period. The zero proprietary period policy also applies to our cycle 8 Spitzer observations. All HST and Spitzer images may be downloaded immediately after they are obtained via the MAST and IRSA archives, respectively. The CLASH collaboration is producing high-level science products that will be publicly distributed via MAST's Treasury Program archive site. ${ }^{26}$ This paper includes the first public release of a CLASH source list for the cluster MACS1149.6 + 2223 as well as uniformly re-derived X-ray properties for the 25 clusters in the sample. The CLASH HLSP include co-added and mosaicked images, weight maps, inverse variance maps, high-resolution color images, and source lists. At the end of the survey we will also release the corresponding registered X-ray surface brightness images, co-added Spitzer Space Telescope images, co-added SZE data, the calibrated lensing-derived mass and source magnification maps, the source lists with all photometric redshift information, SN coordinates and their light curves and grism spectra, and the associated spectroscopic redshift catalogs. The CLASH data provide a vast legacy archive for studies of the formation and evolution of cosmic structure.

We are especially grateful to our program coordinator Beth Perrillo for her expert assistance in implementing the HST observations in this program. We thank Jay Anderson and Norman Grogin for providing the ACS CTE and bias-striping correction algorithms used in our data pipeline. Finally, we are indebted to the hundreds of people who have labored many years to plan, develop, manufacture, install, repair, and calibrate the WFC 3 and ACS instruments as well as to all those who maintain and operate the Hubble Space Telescope.

The CLASH Multi-Cycle Treasury Program (GO-12065) is based on observations made with the NASA/ESA Hubble Space Telescope. The Space Telescope Science Institute is operated by the Association of Universities for Research in Astronomy, Inc., under NASA contract NAS 5-26555. ACS was developed under NASA contract NAS 5-32864.

This research is supported in part by NASA grant HSTGO-12065.01-A, the Israel Science Foundation, the BadenWuerttemberg Foundation, the German Science Foundation (Transregio TR 33), Spanish MICINN grant AYA2010-22111C03-00, funding from the Junta de Andaluca Proyecto de Excelencia NBL2003, INAF contracts ASI-INAF I/009/10/0, ASIINAF I/023/05/0, ASI-INAF I/088/06/0, PRIN INAF 2009, and PRIN INAF 2010, NSF CAREER grant AST-0847157, the UK's STFC, the Royal Society, the Wolfson Foundation, and National Science Council of Taiwan grant NSC97-2112-M001-020-MY3. A.Z. acknowledges support by the John Bahcall excellence prize. L.I. acknowledges support from a Conicyt FONDAP/BASAL grant. P.R. and S.S. acknowledge support

\footnotetext{
26 http://archive.stsci.edu/hst/tall.html
}

from the DFG cluster of excellence Origin and Structure of the Universe program.

\section{REFERENCES}

Abell, G. O. 1958, ApJS, 3, 211

Abell, G. O., Corwin, H. G., Jr., \& Olowin, R. P. 1989, ApJS, 70, 1 Allen, S. W., Rapetti, D. A., Schmidt, R. W., et al. 2008, MNRAS, 383, 879 Anders, E., \& Grevesse, N. 1989, Geochim. Cosmochim. Acta, 53, 197 Anderson, J., \& Bedin, L. R. 2010, PASP, 122, 1035

Beckwith, S. V. W., Stiavelli, M., Koekemoer, A. M., et al. 2006, AJ, 132, 1729

Bell, E. F., Wolf, C., Meisenheimer, K., et al. 2004, ApJ, 608, 752

Benítez, N. 2000, ApJ, 536, 571

Benítez, N., Ford, H., Bouwens, R., et al. 2004, ApJS, 150, 1

Benítez, N., Moles, M., Aguerri, J. A. L., et al. 2009, ApJ, 692, L5

Bertin, E., \& Arnouts, S. 1996, A\&AS, 117, 393

Blakeslee, J. P., Anderson, K. R., Meurer, G. R., Benítez, N., \& Magee, D. 2003, in ASP Conf. Ser. 295, Astronomical Data Analysis Software and Systems XII, ed. H. E. Payne (San Francisco, CA: ASP), 257

Bouwens, R. J., Illingworth, G. D., Blakeslee, J. P., \& Franx, M. 2006, ApJ, 653,53

Bouwens, R. J., Illingworth, G. D., Bradley, L. D., et al. 2009, ApJ, 690, 1764 Bouwens, R. J., Illingworth, G. D., Franx, M., \& Ford, H. 2007, ApJ, 670, 928 Bouwens, R. J., Illingworth, G. D., Franx, M., \& Ford, H. 2008, ApJ, 686, 230 Bouwens, R. J., Illingworth, G. D., Oesch, P. A., et al. 2011, ApJ, 737, 90

Bradley, L. D., Bouwens, R. J., Ford, H. C., et al. 2008, ApJ, 678, 647 Bradley, L. D., Bouwens, R. J., Zitrin, A., et al. 2012, ApJ, 747, 3 Brandt, T. D., Tojeiro, R., Aubourg, É., et al. 2010, AJ, 140, 804 Brinchmann, J., \& Ellis, R. S. 2000, ApJ, 536, L77

Broadhurst, T. J., \& Barkana, R. 2008, MNRAS, 390, 1647

Broadhurst, T., Benítez, N., Coe, D., et al. 2005, ApJ, 621, 53

Broadhurst, T., Umetsu, K., Medezinski, E., Oguri, M., \& Rephaeli, Y. 2008, ApJ, 685, L9

Bunker, A. J., Wilkins, S., Ellis, R. S., et al. 2010, MNRAS, 409, 855

Buote, D. A., Gastaldello, F., Humphrey, P. J., et al. 2007, ApJ, 664, 123

Capak, P., Mobasher, B., Scoville, N. Z., et al. 2011, ApJ, 730, 68

Castellano, M., Fontana, A., Paris, D., et al. 2010, A\&A, 524, A28

Cavagnolo, K. W., Donahue, M., Voit, G. M., \& Sun, M. 2008, ApJ, 682, 821

Clowe, D., Bradač, M., Gonzalez, A. H., et al. 2006, ApJ, 648, L109

Coe, D. 2010, arXiv:1005.0411

Coe, D., Benítez, N., Broadhurst, T., Moustakas, L., \& Ford, H. 2010, ApJ, 723, 1678

Coe, D., Benítez, N., Sánchez, S. F., et al. 2006, AJ, 132, 926

Comerford, J. M., \& Natarajan, P. 2007, MNRAS, 379, 190

Cowie, L. L., Hu, E. M., \& Songaila, A. 1995, Nature, 377, 603

Cowie, L. L., Songaila, A., Hu, E. M., \& Cohen, J. G. 1996, AJ, 112, 839

Dahlen, T., Strolger, L.-G., \& Riess, A. G. 2008, ApJ, 681, 462

Dahlen, T., Strolger, L.-G., Riess, A. G., et al. 2004, ApJ, 613, 189

Dekel, A., \& Birnboim, Y. 2006, MNRAS, 368, 2

Diaferio, A., \& Geller, M. J. 1997, ApJ, 481, 633

Dicker, S. R., Korngut, P. M., Mason, B. S., et al. 2008, Proc. SPIE, 702005-702005-9

Dicker, S. R., Mason, B. S., Korngut, P. M., et al. 2009, ApJ, 705, 226

Dickey, J. M., \& Lockman, F. J. 1990, ARA\&A, 28, 215

Domínguez, I., Höflich, P., \& Straniero, O. 2001, ApJ, 557, 279

Dressel, L. 2010, Wide Field Camera 3 Instrument Handbook, Version 3 http://www.stsci.edu/hst/wfc3/documents/handbooks/currentIHB/wfc3 cover.html

Duffy, A. R., Schaye, J., Kay, S. T., \& Dalla Vecchia, C. 2008, MNRAS, 390, L64

Duffy, A. R., Schaye, J., Kay, S. T., et al. 2010, MNRAS, 405, 2161

Ebeling, H., Barrett, E., Donovan, D., et al. 2007, ApJ, 661, L33

Ebeling, H., Edge, A. C., \& Henry, J. P. 2001, ApJ, 553, 668

Ebeling, H., Edge, A. C., Mantz, A., et al. 2010, MNRAS, 407, 83

Einasto, J. 1965, Trudy Inst. Astroz. Alma-Ata, 51, 87

Eisenhardt, P. R. M., Brodwin, M., Gonzalez, A. H., et al. 2008, ApJ, 684, 905

Elíasdóttir, Á., Limousin, M., Richard, J., et al. 2007, arXiv:0710.5636

Elmegreen, D. M., Elmegreen, B. G., \& Hirst, A. C. 2004a, ApJ, 604, L21

Elmegreen, D. M., Elmegreen, B. G., \& Sheets, C. M. 2004b, ApJ, 603, 74

Ettori, S., Gastaldello, F., Leccardi, A., et al. 2010, A\&A, 524, A68

Faber, S. M., Trager, S. C., Gonzalez, J. J., \& Worthey, G. 1995, in IAU Symp. 164, Stellar Populations, ed. P. C. van der Kruit \& G. Gilmore (Cambridge: Cambridge Univ. Press), 249

Faber, S. M., Willmer, C. N. A., Wolf, C., et al. 2007, ApJ, 665, 265

Fazio, G. G., Hora, J. L., Allen, L. E., et al. 2004, ApJS, 154, 10 
Fedeli, C., \& Bartelmann, M. 2007, A\&A, 461, 49

Fioc, M., \& Rocca-Volmerange, B. 1997, A\&A, 326, 950

Foley, R. J., Andersson, K., Bazin, G., et al. 2011, ApJ, 731, 86

Ford, H. C., Clampin, M., Hartig, G. F., et al. 2003, Proc. SPIE, 4854, 81

Francis, M. J., Lewis, G. F., \& Linder, E. V. 2009, MNRAS, 394, 605

Garmire, G. P., Bautz, M. W., Ford, P. G., Nousek, J. A., \& Ricker, G. R., Jr. 2003, Proc. SPIE, 4851, 28

Genzel, R., Newman, S., Jones, T., et al. 2011, ApJ, 733, 101

Giavalisco, M., Ferguson, H. C., Koekemoer, A. M., et al. 2004, ApJ, 600, L93

Gilmour, R., Best, P., \& Almaini, O. 2009, MNRAS, 392, 1509

Gobat, R., Daddi, E., Onodera, M., et al. 2011, A\&A, 526, A133

Golwala, S., Ameglio, S., Pierpaoli, E., \& Sayers, J. 2009, in ASP Conf. Ser. 417, Submillimeter Astrophysics and Technology: A Symposium Honoring Thomas G. Phillips, ed. D. C. Lis, J. E. Vaillancourt, P. F. Goldsmith, T. A. Bell, N. Z. Scoville, \& J. Zmuidzinas (San Francisco, CA: ASP), 311 Graur, O., Poznanski, D., Maoz, D., et al. 2011, MNRAS, 417, 916 Grogin, N. A., Kocevski, D. D., Faber, S. M., et al. 2011, ApJS, 197, 35 Grossi, M., \& Springel, V. 2009, MNRAS, 394, 1559

Guzman, R., Gallego, J., Koo, D. C., et al. 1997, ApJ, 489, 559

Hennawi, J. F., Dalal, N., Bode, P., \& Ostriker, J. P. 2007, ApJ, 654, 714

Hoekstra, H., Yee, H. K. C., \& Gladders, M. D. 2002, ApJ, 577, 595

Horiuchi, S., \& Beacom, J. F. 2010, ApJ, 723, 329

Huang, X., Morokuma, T., Fakhouri, H. K., et al. 2009, ApJ, 707, L12

Iben, I., Jr., \& Tutukov, A. V. 1984, ApJS, 54, 335

Ilbert, O., Arnouts, S., McCracken, H. J., et al. 2006, A\&A, 457, 841

Ilbert, O., Capak, P., Salvato, M., et al. 2009, ApJ, 690, 1236

Iye, M., Ota, K., Kashikawa, N., et al. 2006, Nature, 443, 186

Jee, M. J., Dawson, K. S., Hoekstra, H., et al. 2011, ApJ, 737, 59

Jee, M. J., Ford, H. C., Illingworth, G. D., et al. 2007, ApJ, 661, 728

Jee, M. J., Rosati, P., Ford, H. C., et al. 2009, ApJ, 704, 672

Johnston, D. E., Sheldon, E. S., Wechsler, R. H., et al. 2007, arXiv:0709.1159

Juneau, S., Glazebrook, K., Crampton, D., et al. 2005, ApJ, 619, L135

Kereš, D., Katz, N., Weinberg, D. H., \& Davé, R. 2005, MNRAS, 363, 2

Khochfar, S., \& Burkert, A. 2003, ApJ, 597, L117

Kimble, R. A., MacKenty, J. W., O'Connell, R. W., \& Townsend, J. A. 2008, Proc. SPIE, 70101E-70101E-12

King, L., \& Mead, J. 2011, MNRAS, 416, 2539

Kneib, J., Ellis, R. S., Santos, M. R., \& Richard, J. 2004, ApJ, 607, 697

Koekemoer, A. M., Faber, S. M., Ferguson, H. C., et al. 2011, ApJS, 197, 36

Koekemoer, A. M., Fruchter, A. S., Hook, R. N., \& Hack, W. 2002, in The 2002 HST Calibration Workshop: Hubble after the Installation of the ACS and the NICMOS Cooling System, ed. S. Arribas, A. Koekemoer, \& B. Whitmore (Baltimore, MD: Space Telescope Science Institute), 337

Komatsu, E., Smith, K. M., Dunkley, J., et al. 2011, ApJS, 192, 18

Kriek, M., van Dokkum, P. G., Labbé, I., et al. 2009, ApJ, 700, 221

Lehnert, M. D., Nesvadba, N. P. H., Cuby, J.-G., et al. 2010, Nature, 467, 940

Lemze, D., Barkana, R., Broadhurst, T. J., \& Rephaeli, Y. 2008, MNRAS, 386, 1092

Lemze, D., Sadeh, S., \& Rephaeli, Y. 2009, MNRAS, 397, 1876

Limousin, M., Richard, J., Jullo, E., et al. 2007, ApJ, 668, 643

Limousin, M., Richard, J., Kneib, J.-P., et al. 2008, A\&A, 489, 23

Longhetti, M., Saracco, P., Severgnini, P., et al. 2007, MNRAS, 374, 614

Macciò, A. V., Dutton, A. A., \& van den Bosch, F. C. 2008, MNRAS, 391, 1940

Maizy, A., Richard, J., de Leo, M. A., Pelló, R., \& Kneib, J. P. 2010, A\&A, 509 A105

Mandelbaum, R., Seljak, U., \& Hirata, C. M. 2008, J. Cosmol. Astropart. Phys., JCAP08(2008)006

Mannucci, F., Della Valle, M., \& Panagia, N. 2006, MNRAS, 370, 773

Mantz, A., Allen, S. W., Rapetti, D., \& Ebeling, H. 2010, MNRAS, 406, 1759

Maoz, D., \& Badenes, C. 2010, MNRAS, 407, 1314

Maoz, D., Mannucci, F., Li, W., et al. 2011, MNRAS, 412, 1508

Maoz, D., Sharon, K., \& Gal-Yam, A. 2010, ApJ, 722, 1879

Markevitch, M. 1998, ApJ, 504, 27

Maughan, B. J., Jones, C., Forman, W., \& Van Speybroeck, L. 2008, ApJS, 174, 117

McLure, R. J., Dunlop, J. S., Cirasuolo, M., et al. 2010, MNRAS, 403, 960

Mead, J. M. G., King, L. J., Sijacki, D., et al. 2010, MNRAS, 406, 434

Medezinski, E., Broadhurst, T., Umetsu, K., et al. 2007, ApJ, 663, 717

Medezinski, E., Broadhurst, T., Umetsu, K., et al. 2010, MNRAS, 405, 257

Meneghetti, M., Fedeli, C., Pace, F., Gottlöber, S., \& Yepes, G. 2010, A\&A, 519, A90

Meneghetti, M., Fedeli, C., Zitrin, A., et al. 2011, A\&A, 530, A17

Miralda-Escude, J. 1998, ApJ, 501, 15
Miyazaki, S., Sekiguchi, M., Imi, K., et al. 1998, Proc. SPIE, 3355, 363

Molnar, S. M., Chiu, I., Umetsu, K., et al. 2010, ApJ, 724, L1

Morandi, A., Pedersen, K., \& Limousin, M. 2011, ApJ, 729, 37

Navarro, J. F., Frenk, C. S., \& White, S. D. M. 1996, ApJ, 462, 563

Navarro, J. F., Frenk, C. S., \& White, S. D. M. 1997, ApJ, 490, 493

Navarro, J. F., Hayashi, E., Power, C., et al. 2004, MNRAS, 349, 1039

Navarro, J. F., Ludlow, A., Springel, V., et al. 2010, MNRAS, 402, 21

Neto, A. F., Gao, L., Bett, P., et al. 2007, MNRAS, 381, 1450

Newman, A. B., Treu, T., Ellis, R. S., \& Sand, D. J. 2011, ApJ, 728, L39

Newman, A. B., Treu, T., Ellis, R. S., et al. 2009, ApJ, 706, 1078

Nomoto, K. 1982, ApJ, 253, 798

Oesch, P. A., Bouwens, R. J., Carollo, C. M., et al. 2010a, ApJ, 709, L21

Oesch, P. A., Bouwens, R. J., Illingworth, G. D., et al. 2010b, ApJ, 709, L16

Oguri, M., \& Blandford, R. D. 2009, MNRAS, 392, 930

Oguri, M., \& Hamana, T. 2011, MNRAS, 414, 1851

Oguri, M., Hennawi, J. F., Gladders, M. D., et al. 2009, ApJ, 699, 1038

Okabe, N., Takada, M., Umetsu, K., Futamase, T., \& Smith, G. P. 2010a, PASJ, 62,811

Okabe, N., Zhang, Y., Finoguenov, A., et al. 2010b, ApJ, 721, 875

Oke, J. B. 1974, ApJS, 27, 21

Ono, Y., Ouchi, M., Mobasher, B., et al. 2012, ApJ, 744, 83

Oser, L., Ostriker, J. P., Naab, T., Johansson, P. H., \& Burkert, A. 2010, ApJ, 725, 2312

Papovich, C., Momcheva, I., Willmer, C. N. A., et al. 2010, ApJ, 716, 1503

Perlmutter, S., Aldering, G., Goldhaber, G., et al. 1999, ApJ, 517, 565

Prada, F., Klypin, A. A., Cuesta, A. J., Betancort-Rijo, J. E., \& Primack, J. 2011, arXiv: 1104.5130

Pratt, G. W., Croston, J. H., Arnaud, M., \& Böhringer, H. 2009, A\&A, 498, 361

Pritchet, C. J., Howell, D. A., \& Sullivan, M. 2008, ApJ, 683, L25

Proctor, R. N., \& Sansom, A. E. 2002, MNRAS, 333, 517

Rafelski, M., Wolfe, A. M., Cooke, J., et al. 2009, ApJ, 703, 2033

Richard, J., Kneib, J., Ebeling, H., et al. 2011, MNRAS, 414, L31

Richard, J., Smith, G. P., Kneib, J., et al. 2010, MNRAS, 404, 325

Riess, A. G., Filippenko, A. V., Challis, P., et al. 1998, AJ, 116, 1009

Riess, A. G., \& Livio, M. 2006, ApJ, 648, 884

Riess, A. G., Macri, L., Casertano, S., et al. 2011, ApJ, 730, 119

Riess, A. G., Strolger, L., Casertano, S., et al. 2007, ApJ, 659, 98

Riess, A. G., Strolger, L.-G., Tonry, J., et al. 2004, ApJ, 607, 665

Rines, K., \& Diaferio, A. 2006, AJ, 132, 1275

Rines, K., Geller, M. J., \& Diaferio, A. 2010, ApJ, 715, L180

Rosati, P., Tozzi, P., Gobat, R., et al. 2009, A\&A, 508, 583

Sadeh, S., \& Rephaeli, Y. 2008, MNRAS, 388, 1759

Saha, P., \& Read, J. I. 2009, ApJ, 690, 154

Salvaterra, R., Della Valle, M., Campana, S., et al. 2009, Nature, 461, 1258

Sand, D. J., Treu, T., Ellis, R. S., Smith, G. P., \& Kneib, J. 2008, ApJ, 674, 711

Sand, D. J., Treu, T., Smith, G. P., \& Ellis, R. S. 2004, ApJ, 604, 88

Sayers, J., Golwala, S. R., Ameglio, S., \& Pierpaoli, E. 2011, ApJ, 728, 39

Schenker, M. A., Stark, D. P., Ellis, R. S., et al. 2012, ApJ, 744, 179

Schlegel, D. J., Finkbeiner, D. P., \& Davis, M. 1998, ApJ, 500, 525

Schmidt, R. W., \& Allen, S. W. 2007, MNRAS, 379, 209

Schwope, A. D., Lamer, G., de Hoon, A., et al. 2010, A\&A, 513, L10

Seidel, G., \& Bartelmann, M. 2007, A\&A, 472, 341

Sereno, M., Jetzer, P., \& Lubini, M. 2010, MNRAS, 403, 2077

Sérsic, J. L. 1963, Boletin de la Asociacion Argentina de Astronomia La Plata Argentina, 6, 41

Sirianni, M., De Marchi, G., Gilliland, R., et al. 2003, BAAS, 35, 722

Smith, G. P., Kneib, J.-P., Smail, I., et al. 2005, MNRAS, 359, 417

Stanford, S. A., Romer, A. K., Sabirli, K., et al. 2006, ApJ, 646, L13

Strüder, L., Briel, U., Dennerl, K., et al. 2001, A\&A, 365, L18

Su, J., Stiavelli, M., Oesch, P., et al. 2011, ApJ, 738, 123

Tanvir, N. R., Fox, D. B., Levan, A. J., et al. 2009, Nature, 461, 1254

Thomas, D., Maraston, C., Bender, R., \& Mendes de Oliveira, C. 2005, ApJ, 621,673

Totani, T., Morokuma, T., Oda, T., Doi, M., \& Yasuda, N. 2008, PASJ, 60, 1327

Trujillo, I., Feulner, G., Goranova, Y., et al. 2006, MNRAS, 373, L36

Turner, M. J. L., Abbey, A., Arnaud, M., et al. 2001, A\&A, 365, L27

Umetsu, K., Birkinshaw, M., Liu, G., et al. 2009, ApJ, 694, 1643

Umetsu, K., Broadhurst, T., Zitrin, A., Medezinski, E., \& Hsu, L. 2011a, ApJ, 729,127

Umetsu, K., Broadhurst, T., Zitrin, A., et al. 2011b, ApJ, 738, 41

Umetsu, K., Medezinski, E., Broadhurst, T., Zitrin, A., Okabe, N., Hsieh, B., \& Molnar, S. M. 2010, ApJ, 714, 1470

van den Bergh, S. 1996, AJ, 112, 2634 
van Dokkum, P. G. 2008, ApJ, 674, 29

Webbink, R. F. 1984, ApJ, 277, 355

Werner, M. W., Roellig, T. L., Low, F. J., et al. 2004, ApJS, 154, 1

Whelan, J., \& Iben, I., Jr. 1973, ApJ, 186, 1007

Worthey, G. 1996, in ASP Conf. Ser. 98, From Stars to Galaxies: the Impact of Stellar Physics on Galaxy Evolution, ed. C. Leitherer, U. Fritze-vonAlvensleben, \& J. Huchra (San Francisco, CA: ASP), 467

Wuyts, S., Labbé, I., Schreiber, N. M. F., et al. 2008, ApJ, 682, 985

Zhang, Y., Okabe, N., Finoguenov, A., et al. 2010, ApJ, 711, 1033

Zheng, W., Bradley, L. D., Bouwens, R. J., et al. 2009, ApJ, 697, 1907

Zitrin, A., Broadhurst, T., Barkana, R., Rephaeli, Y., \& Benítez, N. 2011a, MNRAS, 410, 1939

Zitrin, A., Broadhurst, T., Bartelmann, M., et al. 2011b, arXiv:1105.2295

Zitrin, A., Broadhurst, T., Coe, D., et al. 2011c, MNRAS, 413, 1753

Zitrin, A., Broadhurst, T., Umetsu, K., et al. 2009, MNRAS, 396, 1985

Zitrin, A., Broadhurst, T., Umetsu, K., et al. 2010, MNRAS, 408, 1916 